\author{
RESEARCH ARTICLE \\ 10.1029/2020JE006418 \\ Key Points: \\ - The signal from induction that \\ accounts for adiabatic ocean \\ temperatures is distinct from \\ induction based on uniform \\ conductivity \\ - Motional induction due to thermal \\ convection in the satellite oceans \\ may be significant \\ - Material properties and motional \\ induction modeling are needed \\ to obtain ocean composition from \\ magnetic induction
}

Supporting Information:

- Supporting Information S1

Correspondence to:

S. D. Vance,

svance@jpl.caltech.edu

Citation:

Vance, S. D., Styczinski, M. J., Bills, B. G., Cochrane, C. J., Soderlund, K. M., Gómez-Pérez, N., \& Paty, C. (2021)

Magnetic induction responses of Jupiter's ocean moons including effects from adiabatic convection. Journal of Geophysical Research: Planets, 126, e2020JE006418. https://doi. org/10.1029/2020JE006418

Received 25 FEB 2020

Accepted 13 DEC 2020

Author Contributions:

Conceptualization: S. D. Vance, M. J. Styczinski, B. G. Bills, C. J. Cochrane, K. M. Soderlund

Data curation: S. D. Vance, M. J.

Styczinski

Formal analysis: M. J. Styczinski, B. G. Bills, C. J. Cochrane, K. M. Soderlund Investigation: $\mathrm{S}$. D. Vance Methodology: M. J. Styczinski, B. G. Bills, C. J. Cochrane, K. M. Soderlund, N. Gómez-Pérez

Project administration: S. D. Vance

Resources: C. J. Cochrane, K. M.

Soderlund

Software: S. D. Vance, M. J. Styczinski, B. G. Bills

Supervision: S. D. Vance

Writing - original draft: S. D. Vance,

B. G. Bills, K. M. Soderlund

(C) 2020. American Geophysical Union. All Rights Reserved.

\section{Magnetic Induction Responses of Jupiter's Ocean Moons Including Effects From Adiabatic Convection}

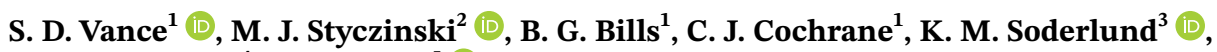 \\ N. Gómez-Pérez ${ }^{4}$ and C. Paty ${ }^{5}$ (D) \\ ${ }^{1}$ Jet Propulsion Laboratory, California Institute of Technology, Pasadena, CA, USA, ${ }^{2}$ Department of Physics, University \\ of Washington, Seattle, WA, USA, ${ }^{3}$ Institute for Geophysics, John A. \& Katherine G. Jackson School of Geosciences, \\ The University of Texas at Austin, Austin, TX, USA, ${ }^{4}$ British Geological Survey, Edinburgh, UK, ${ }^{5}$ Department of Earth \\ Sciences, University of Oregon, Eugene, OR, USA
}

Abstract Prior analyses of oceanic magnetic induction within Jupiter's large icy moons have assumed uniform electrical conductivity. However, the phase and amplitude responses of the induced fields will be influenced by the natural depth-dependence of the electrical conductivity. Here, we examine the amplitudes and phase delays for magnetic diffusion in modeled oceans of Europa, Ganymede, and Callisto. For spherically symmetric configurations, we consider thermodynamically consistent interior structures that include realistic electrical conductivity along the oceans' adiabatic temperature profiles. Conductances depend strongly on salinity, especially in the large moons. The induction responses of the adiabatic profiles differ from those of oceans with uniform conductivity set to values at the iceocean interface, or to the mean values of the adiabatic profile, by more than $10 \%$ for some signals. We also consider motionally induced magnetic fields generated by convective fluid motions within the oceans, which might optimistically be used to infer ocean flows or, pessimistically, act to bias the ocean conductivity inversions. Our upper-bound scaling estimates suggest this effect may be important at Europa and Ganymede, with a negligible contribution at Callisto. Based on end-member ocean compositions, we quantify the magnetic induction signals that might be used to infer the oxidation state of Europa's ocean and to investigate stable liquids under high-pressure ices in Ganymede and Callisto. Fully exploring this parameter space for the sake of planned missions requires thermodynamic and electrical conductivity measurements in fluids at low temperature and to high-salinity and pressure as well as modeling of motional induction responses.

Plain Language Summary Oscillations in a planet's magnetic fields can create magnetic signals within its companion moons if those moons have salty oceans under their icy surfaces. Fluid currents within those oceans can also create magnetic fields. Spacecraft investigating those oceans can measure such fields and thereby learn about the properties of the oceans. We compute possible magnetic properties for Jupiter's ocean moons_Europa, Ganymede, and Callisto—using available chemical data and electrical conductivity. Previous work has also computed these properties, but our methods allow us to account for how the electrical properties vary with depth due to pressure and temperature. We also model ocean currents. We find that the depth-dependence of electrical conductivity affects the predicted magnetic fields more than $1 \mathrm{nT}$ as compared with the typical assumption of a uniform conductivity. This is important because the planned Europa Clipper and JUpiter ICy moons Explorer (JUICE) missions will measure magnetic fields at these moons with roughly $1 \mathrm{nT}$ precision. For Europa, we examine seawater $(\mathrm{NaCl})$ and $\mathrm{MgSO}_{4}$ ocean compositions linked to more hydrogen-rich or oxygen-rich scenarios. With additional constraints on ice shell and ocean thickness from other spacecraft measurements, magnetic measurements may be a key tool for determining the composition of Europa's ocean, and thus its chemical evolution through time.

\section{Introduction}

The jovian system is of particular interest for studying magnetic induction in icy ocean worlds. Jupiter has a strong magnetic field whose dipole axis is tilted $9.6^{\circ}$ with respect to its rotation axis (Acuna \& Ness, 1976), while the orbits of the Galilean moons lie very nearly in the equatorial plane of Jupiter. This means that Jupiter's magnetic field varies in time at the orbital positions of the satellites. Also, the outer layers of the 
Writing - review \& editing: S. D. Vance, B. G. Bills, C. J. Cochrane, K. M. Soderlund, C. Paty satellites themselves are believed to consist mainly of water ice at the surface, underlain by salty oceans. Brines are good conductors, while ice is a significant insulator.

Magnetic induction from Jupiter's diurnal signal sensed by the Galileo mission provides the most compelling direct observational evidence for the existence of oceans within Europa and Ganymede (Hand \& Chyba, 2007; Khurana et al., 1998; Khurana \& Russell, 2009; Kivelson et al., 2000; Saur, Strobel, \& Neubauer, 1998; Schilling, Neubauer, \& Saur, 2007). The case has also been made for an induction response from an ocean in Callisto (Zimmer, Khurana, \& Kivelson, 2000), but this interpretation may be clouded by interactions with the peak of the jovian current sheet and by ionospheric interactions (Hartkorn \& Saur, 2017; Liuzzo et al, 2015).

Longer-period signals penetrate more deeply, as penetration of the magnetic field into the interior is a diffusive process. It is convenient that the skin depths at the dominant periods of variation experienced by Europa, Ganymede, and Callisto are comparable to the expected ocean depths, which makes it possible to probe the properties of their oceans using magnetic induction (Saur et al., 2009). The spectrum of frequencies driving induced magnetic responses includes not just the orbits of the Galilean satellites and the rotation of Jupiter's tilted dipole field, but also their harmonics and natural oscillations of the magnetospheric structure relative to the satellites' orbital locations (Seufert et al., 2011). Electrical conductivity structure within the subsurface oceans-for example, from convective adiabatic temperature gradients (Vance et al., 2018) and stratification (Vance \& Goodman, 2009)—will affect the induction response at these frequencies.

Further variations in the magnetic fields arise from the motion of the moons about Jupiter. Perturbations to the orbits of the moons arise from multiple sources, including the oblate figure of Jupiter, gravitational interactions with the other satellites, and even from Saturn and the Sun (Lainey et al., 2006; Lieske, 1998). These subtle perturbations introduce additional frequencies of oscillation in the magnetic fields the bodies experience. These additional oscillations, in turn, induce magnetic fields that oscillate on the same time scales. A complete understanding of the dominant frequencies of oscillation is vital to a physically consistent interpretation of spacecraft measurements; for our analysis, we use the NAIF-produced SPICE kernels to obtain the most precise ephemeris data available as they include the orbital perturbations responsible for most magnetic oscillation for the bodies we study.

An additional induced magnetic response may occur in the icy Galilean satellites, arising not from Jupiter's changing magnetic field, but from motions of salty water within the oceans themselves. Such motionally induced magnetic fields are typically neglected because they are expected to be relatively weak. On Earth, ocean currents induce fields on the order of $100 \mathrm{nT}$ in a background field of about 40,000 nT; these fields are observable by space-based magnetometers and have been used to monitor ocean currents (Constable \& Constable, 2004; Tyler et al., 2003). If there are motional induction signals present in the icy Galilean satellites, and if the spatial or temporal structures of these induction signals allow them to be separated from the contributions driven by variations in the jovian magnetic field, it would permit characterization of the ocean flows themselves as has been done for the oceans of Earth (e.g., Chave, 1983; Grayver et al., 2016; Minami, 2017; Tyler et al., 2003). Conversely, if such induced signals are present but the analysis of spacecraft magnetic field measurements does not accommodate that fact, then the recovered electrical conductivity estimates may be biased and inaccurate.

Here, we examine the amplitudes and phase delays for magnetic diffusion in modeled oceans of Europa, Ganymede, and Callisto. For Europa, we focus on whether these responses might reveal not just the ocean's thickness and electrical conductivity, but also the speciation of dissolved salts in the ocean-here either $\mathrm{MgSO}_{4}$ or seawater dominated by $\mathrm{NaCl}$. We restrict our analysis to spherically symmetric configurations, treating interior structures based on self-consistent thermodynamics, which account for variations in electrical conductivity with depth in convective oceans (Vance et al., 2018).

In addition, we consider the generation of motionally induced magnetic fields due to oceanic thermal convection and estimate upper-bound field amplitudes using a scaling analysis. Based on end-member ocean compositions (Zolotov, 2008; Zolotov \& Kargel, 2009), we demonstrate the possibilities for using magnetic induction to infer the oxidation state of Europa's ocean and to identify stable liquid layers under high-pressure ices in Ganymede and Callisto. 
In Section 2, we examine the diffusive induction response of Jupiter's ocean moons. We build on the prior work of Seufert et al. (2011) by including electrical conductivity profiles that follow the adiabatic profiles of pressure and temperature within the ocean of each moon. In Section 3, we describe possible ocean flows due to thermal convection (assuming the models of Soderlund, 2019) and use a scaling relationship to estimate upper bounds for motionally induced magnetic field strengths. In Section 4, we discuss these results and describe the prospects for detecting signals from each. The Supplemental Material includes detailed derivations of the theoretical techniques we use to model the induced magnetic fields, as well as additional results for field components not covered in Sections 2-4.

\section{Diffusive Induction in Jupiter's Ocean Moons}

The complex response to the excitation field $\mathcal{A}_{1}^{e}$ describes the frequency-dependent, normalized amplitude $A=\mid \mathcal{A}_{n}^{e}$ I and phase delay $\phi=-\arg \left(\mathcal{A}_{n}^{e}\right)$ for a uniform excitation field from Jupiter (degree $\left.n=1\right)$. We compute the magnetic induction amplitude and phase delay for a spherically symmetric system with multiple conducting layers. This complex response function is the same as employed by, e.g., Zimmer et al. (2000); Khurana et al. (2002); Seufert et al. (2011), generalized to an arbitrary number of layers and any degree $n$ in the excitation field. A derivation for this solution was first described by Srivastava (1966). Our adapted version from Eckhardt (1963) is provided in the supplement, along with a description of the optimized numerical implementation used in this work. The analytical benchmark described in the supplement builds on recent work by Styczinski et al. (in progress) examining perturbations from spherical symmetry.

\subsection{Spectral Content of the Imposed Magnetic Field Variations}

Temporal variations in the magnetic field occur in the reference frames of Jupiter's satellites. Figure 1 shows time series spectra over the range of periods showing the strongest components for each of Europa, Ganymede, and Callisto, arising from their orbital and synodic periods, as well as beats and harmonics of these periods. Table 1 lists the three main periods (in hr) and the corresponding component fields (in nT). For these analyses, we use body-centric $\phi \Omega$ coordinates $\mathrm{E} \phi \Omega, \mathrm{G} \phi \Omega$, and $\mathrm{C} \phi \Omega$ (e.g. "E-phi-O"; Khurana et al., 2009). In these coordinate systems, $\hat{x}$ is directed along the corotation direction, approximately along the orbital velocity vector, $\hat{y}$ is directed toward the jovian spin axis, approximately toward Jupiter's center of mass, and $\hat{z}$ is directed along the jovian spin axis in a right-handed sense. These coordinate systems are constantly rotating, and remain fixed to center of each satellite. Seufert et al. (2011) determined the time series spectra for the time-varying magnetic perturbations applied to each of the four Galilean moons based on the VIP4 model of Connerney et al. (1998) combined with the jovian current sheet model of Khurana (1997). In contrast, we use the JRM09 Jupiter field model accounting for Juno measurements (Connerney et al., 2018). Paired with this model, we use the current sheet model of Connerney et al. (1981) because the JRM09 model is derived using this current sheet model. Together, the latter two match the Juno measurements well. We compute a time series of the field at the orbital positions of the moons using the NAIF SPICE kernels and 10 years of data sampled at a ten-minute cadence. To determine the primary periods relevant to the diffusive interaction with the satellites, we compute the Fourier transform of the entire data set.

We note that Seufert et al. (2011) also examined the time series spectra of magnetic perturbations from dynamic migration of the jovian magnetopause based on solar wind data from the Ulysses spacecraft, which we do not consider.

The temporal variations in imposed magnetic field at each satellite depend on the orbits of the satellites and the magnetic field of Jupiter. To find them, we compute Jupiter's magnetic field in a Jupiter-centered coordinate system from a spherical harmonic series representation of the magnetic potential (Parkinson, 1983):

$$
\Phi(r, \theta, \phi, t)=R \sum_{n=1}\left(\frac{R}{r}\right)^{n+1} \sum_{m=0}^{n} S_{n, m}(\theta, \varphi) \mathrm{e}^{-i \omega t}
$$

for Jupiter's rotation rate $\omega$ and $R$ the outer radius of the body. The internally generated magnetic field vector is the negative gradient of the scalar potential 

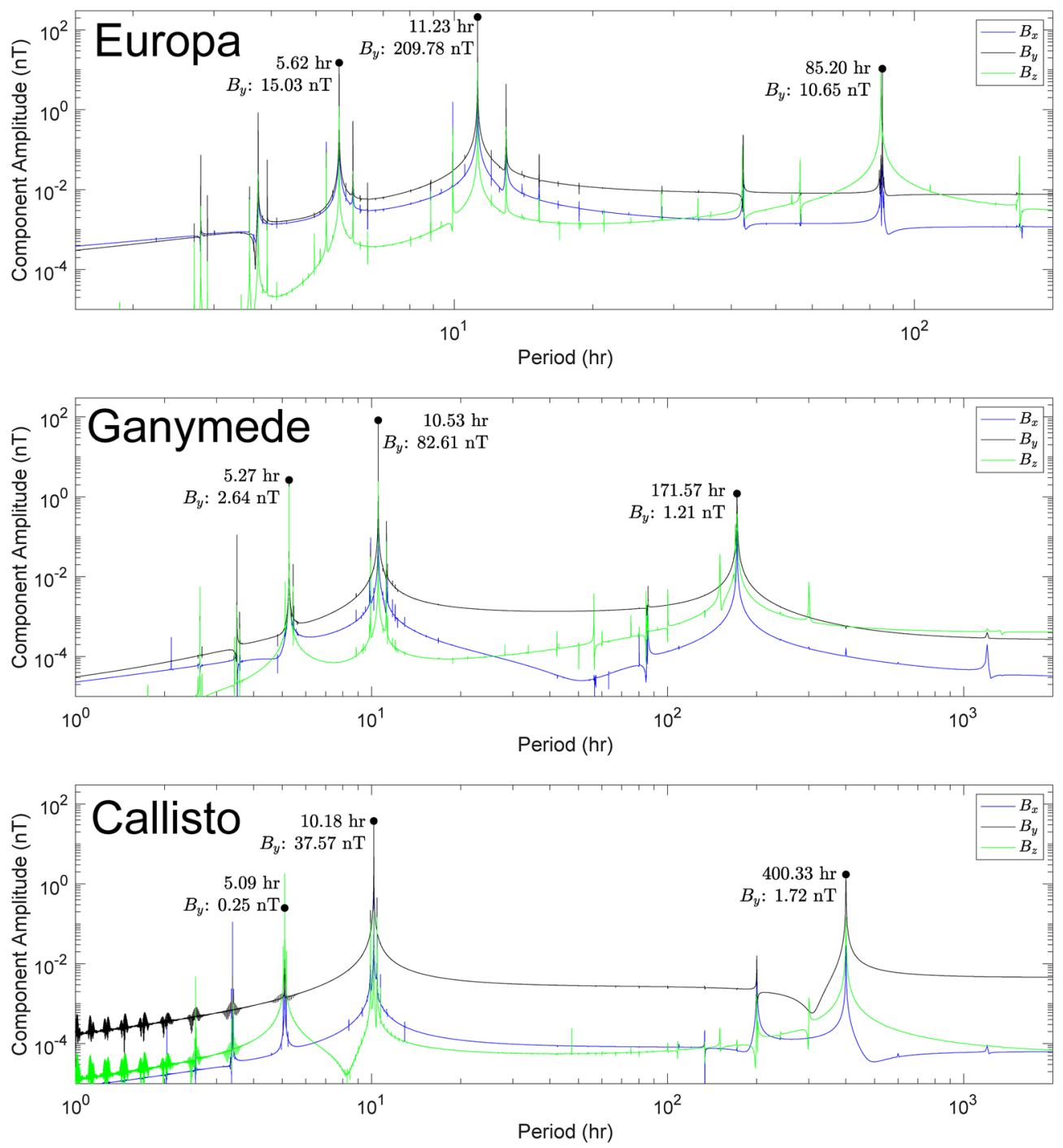

Figure 1. Time series spectra (in hr) for the largest magnetic field oscillations (in nT) experienced by the Galilean moons. Variations in orbital parameters over time introduce magnetic fluctuations at multiple periods in addition to Jupiter's synodic rotation and the satellites' orbits. The coordinate axes are detailed in Section 2.1. Peak values for the main three periods for each moon are provided in Table 1. The input time series is 10 years long; the spectra are sampled with about 500,000 data points in uniform, ten-minute increments.

Table 1

Peak Periods (in h) and Component Field Strengths (in nT) for the Time Series Spectra Shown in Figure 1

\begin{tabular}{|c|c|c|c|c|c|c|c|c|c|}
\hline & \multicolumn{9}{|c|}{ Period (hr) } \\
\hline & \multicolumn{3}{|c|}{$B_{x, y, z}(\mathrm{nT})$} & \multicolumn{3}{|c|}{$B_{x, y, z}(\mathrm{nT})$} & \multicolumn{3}{|c|}{$B_{x, y, z}(\mathrm{nT})$} \\
\hline \multirow[t]{2}{*}{ Europa } & & 5.62 & & & 11.23 & & & 85.20 & \\
\hline & 10.03 & 15.03 & 1.22 & 75.55 & 209.78 & 15.24 & 3.17 & 10.65 & 11.97 \\
\hline \multirow[t]{2}{*}{ Ganymede } & & 5.27 & & & 10.53 & & & 171.57 & \\
\hline & 1.76 & 2.64 & 1.78 & 16.64 & 82.61 & 2.42 & 0.14 & 1.21 & 0.38 \\
\hline \multirow[t]{2}{*}{ Callisto } & & 5.09 & & & 10.18 & & & 400.33 & \\
\hline & 0.17 & 0.25 & 1.82 & 1.31 & 37.57 & 0.20 & 0.03 & 1.72 & 0.14 \\
\hline
\end{tabular}




$$
\mathbf{B}_{\text {int,Jup }}=-\nabla \Phi
$$

The external field including the current systems is

$$
\mathbf{B}_{\text {external }}=\nabla \times \mathbf{A}\left(\rho^{\prime}, z^{\prime}\right) \mathrm{e}^{-i \omega t}
$$

where $\mathbf{A}\left(\rho^{\prime}, z^{\prime}\right)$ is described by the current sheet model of Connerney et al. (1981), $\rho^{\prime}$ and $z^{\prime}$ are radial and axial coordinates in the magnetic equatorial cylindrical coordinate system, and $\omega$ is again Jupiter's rotation rate. The magnetic field applied to the Galilean moons is found by taking the sum of these

$$
\mathbf{B}_{o}=\mathbf{B}_{\text {int,Jup }}+\mathbf{B}_{\text {external }}
$$

Within the conducting portion of the satellites, the net magnetic field $\mathbf{B}$ must satisfy the Helmholtz equation

$$
\nabla^{2} \mathbf{B}=-k^{2} \mathbf{B}
$$

which is a diffusion equation for $\mathbf{B}$. The wavenumber $k$ is a function of the material properties and the angular frequency of oscillation of B within the body (see Section S1):

$$
k=\sqrt{i \omega_{p} \mu_{0} \sigma}
$$

All terms within $\mathbf{B}$ are proportional to an oscillation factor $e^{-i \omega_{p} t}$, where $\omega_{p}$ is the angular frequency of oscillation. Only the largest oscillation amplitudes induce significant diffusive responses.

The diffusive response may be expressed in terms of the normalized excitation amplitude

$$
\mathcal{A}_{n}^{e}=\frac{(n+1)}{n} \frac{B_{i}}{B_{e}}
$$

which is a complex quantity that has the desirable property of ranging from 0 for a nonconducting body to $(1+0 \mathrm{i})$ for a perfect conductor. $B_{i}$ and $B_{e}$ are magnetic potentials for the induced and excitation fields, respectively, outside the moon (see Section $\mathrm{S} 1.1 .2$ ).

The magnetic field $\mathbf{B}_{o}$ applied to the Galilean moons is close to uniform across the body of each satellite, so it is customary to choose $n=1$ in the excitation field. In this case, the potential $B_{e}$ is equal to the amplitude of oscillation of the applied field for a particular angular frequency $\omega_{p}$ and has units of nT. On the surface of the body, at the poles, the diffusive response field is directed opposite the applied field. It oscillates as

$$
B_{\mathrm{dif}, p}(t)=B_{e} \mathcal{A}_{1}^{e} e^{-i \omega_{p} t}
$$

and it has the form of a dipole (see Section S1.3). The measured magnetic field is then the real part of the net field outside the moon

$$
\mathbf{B}_{\text {net }}=\mathbf{B}_{o}+\mathbf{B}_{\mathrm{dif}}
$$

which includes sums over all $n, m$, and $p$. The motionally induced fields discussed in Section 3 add another term to Equation 9. For our full mathematical derivation, see Section S1.

Unique among the satellites in our solar system, Ganymede has an internally generated dynamo field (Kivelson et al., 2002). In the case of this satellite, the analysis of the diffusive field is no different because this intrinsic field does not vary with time in the frame of the body. As with the mean background field applied by Jupiter, the dynamo field from Ganymede simply presents a static offset to magnetometer measurements near the body, and does not appear in the Fourier analysis. The magnitude of this net background field, around $800 \mathrm{nT}$ at Ganymede's surface, is about a factor of two larger than that experienced by Europa (Zimmer et al., 2000) and thus does not present significant additional challenges to measurement precision scaling. 

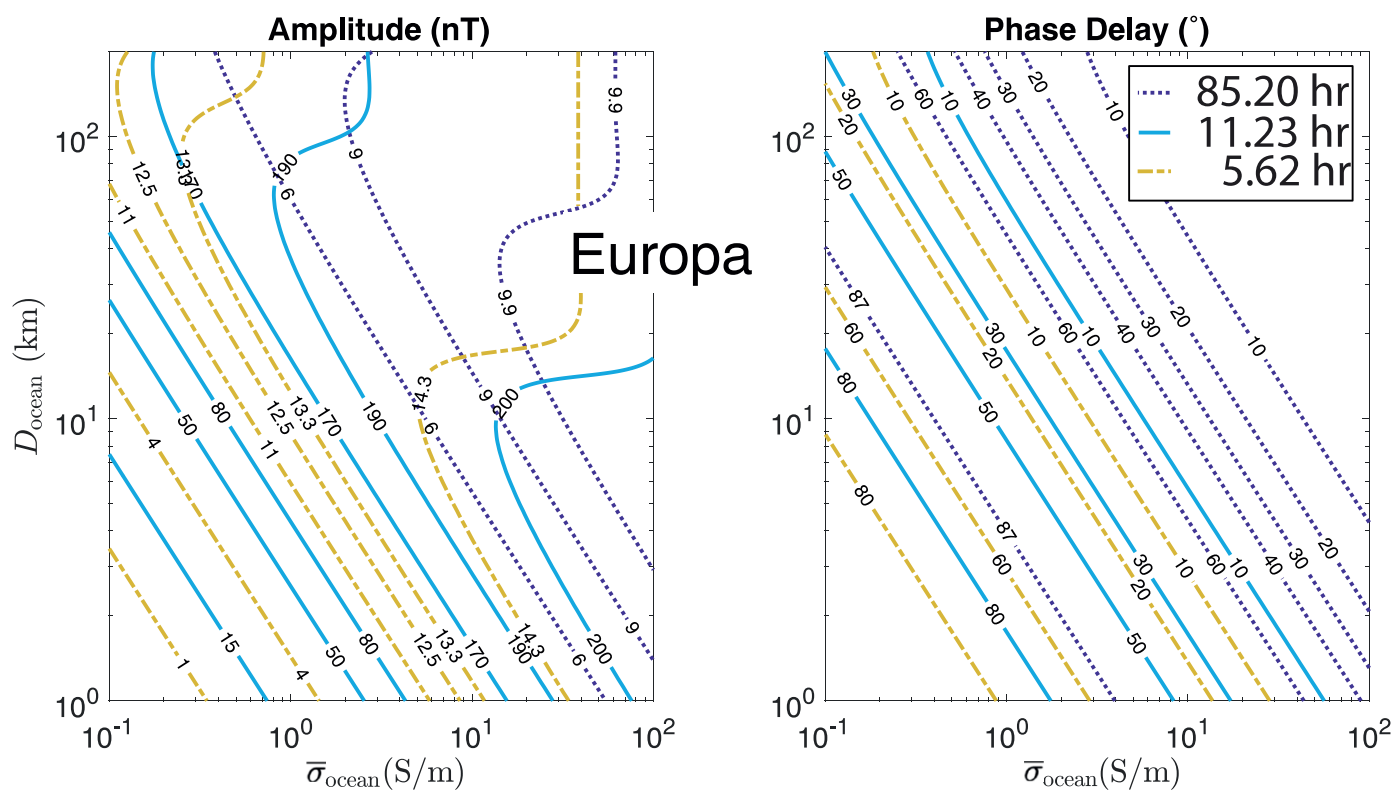

Figure 2. Europa: Contours of the maximum induced field $B_{y}$ components (in nT) and phase delays (in ${ }^{\circ}$ ) at the strongest inducing periods—orbital ( $85.20 \mathrm{~h}$; dotted), Jupiter synodic (11.23 h; solid), and second synodic harmonic (5.62 h; dot-dash) - shown in Figure 1. The assumed, fixed ice thickness of $20 \mathrm{~km}$ and variable seafloor depth yield normalized amplitudes consistent with the previous calculations by Khurana et al. (2002), and phase delays for the synodic frequency matching those described by Zimmer et al. (2000). Unlike in previous work, we scale the amplitudes to the maximum component of the magnetic oscillation the satellite actually experiences at each frequency, which are the largest peaks in Figure 1.

\subsection{Parameter Space of the Diffusive Induction Response}

Previous investigations have considered a continuous parameter space of ocean thickness and conductivity for three-layer models, consisting of a nonconducting mantle (and core), salty ocean, and nonconducting ice (Khurana et al., 2002; Zimmer et al., 2000). A five-layer model, adding an ionosphere and metallic core has also been studied (Schilling, 2006). More recent work by Seufert et al. (2011) has further examined the influence of a metallic core and an ionosphere. None of this prior work required the self-consistency among the ocean temperature and density, composition, ice and ocean thickness, etc., that are the focus of this paper. In this section, we also examine the parameter space of ocean thickness and conductivity for simple three-layer models with uniformly conducting oceans, in the interest of assessing the general range of possible responses and demonstrating consistency between our methods and those used in previous investigations.

Figures 2-4 show contours of the maximum induced magnetic field at the surface as a function of ocean thickness and mean ocean conductivity for each body. These figures show the signals for the three strongest driving periods, which are described in Section 2.1 and shown in Figure 1. Phase delays for the Jupiter synodic frequencies for Europa and Callisto match those described by Zimmer et al. (2000). An ice thickness of $20 \mathrm{~km}$ was set for Europa, consistent with previous calculations by Khurana et al. (2002) (we note that these authors did not specify what ice thickness was used). For both Ganymede and Callisto, $50 \mathrm{~km}$ ice shells were used. In each case, the fixed ice thickness means the seafloor depth varies to accommodate the range of $D_{\text {ocean }}$.

The amplitudes for Europa's orbital and synodic frequencies ( $85.23 \mathrm{~h}$ and $11.23 \mathrm{~h}$ ) match those described by Khurana et al. (2002, 2009). However, these authors scaled the diffusive induction response to an excitation amplitude of 14 and $250 \mathrm{nT}$ for Europa's orbital and synodic periods, respectively; in this work, each contour plot in Figures 2-4 is scaled to the largest relevant peak in the frequency spectrum in Figure 1 . When we instead apply a matching scaling along with a $20 \mathrm{~km}$ ice shell, we generate matching figures.

By choosing a scaling that matches the applied excitation amplitudes, Figures 2-4 indicate the maximum magnetic field components that a magnetometer on the surface of each body would measure at key 

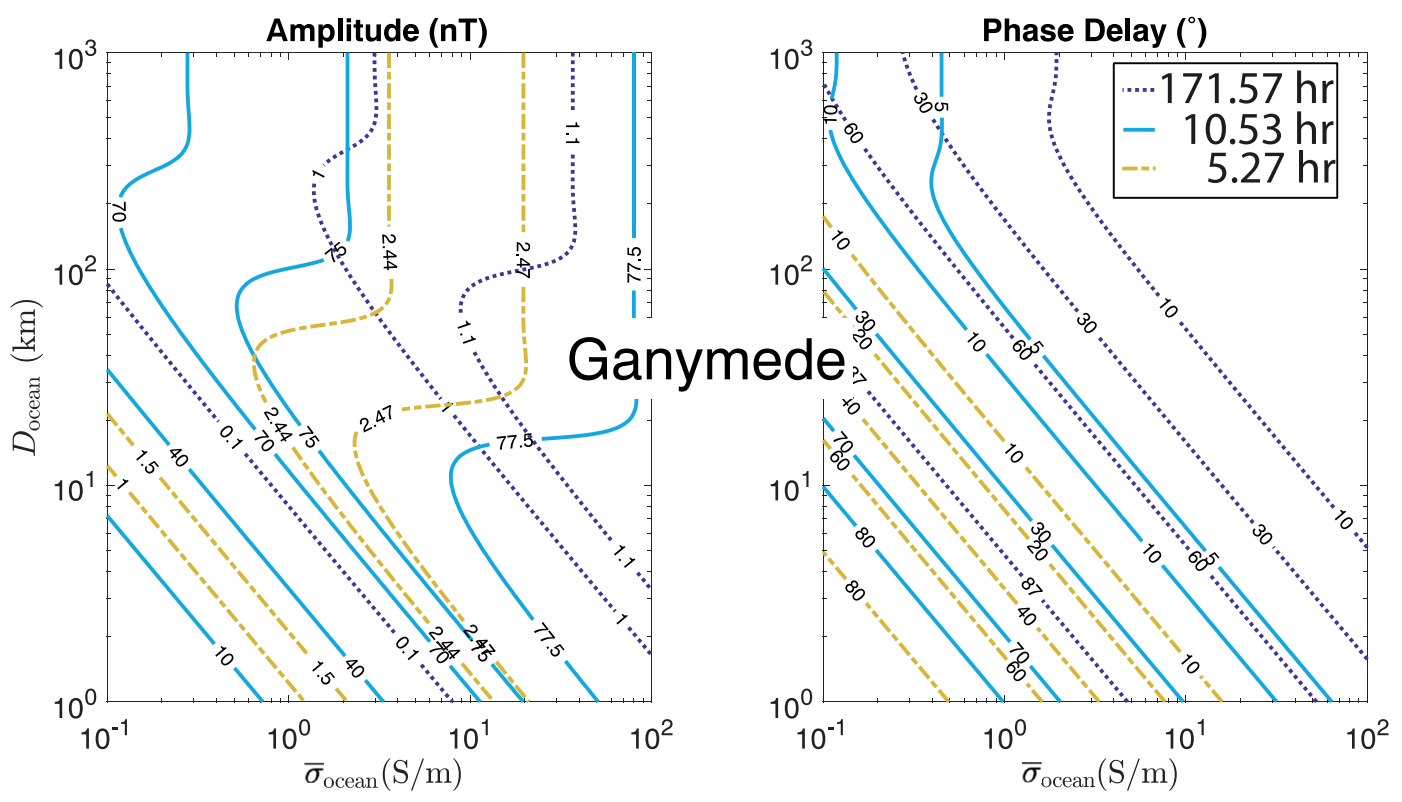

Figure 3. Ganymede: Contours of the maximum induced field $B_{y}$ components (in nT) and phase delays (in ${ }^{\circ}$ ) at the strongest inducing periods—orbital (171.57 h; dotted), Jupiter synodic (10.53 h; solid), and second synodic harmonic $(5.27 \mathrm{~h}$; dot-dash) - shown in Figure 1. The amplitudes and phases for the synodic and orbital periods are comparable to those described by Seufert et al. (2011) for greater ocean conductivities and thicknesses, but these authors model a highly conducting core, which we do not consider. A $50 \mathrm{~km}$ ice shell is assumed at the surface, implying that the seafloor depth varies to accommodate the range of $D_{\text {ocean }}$.
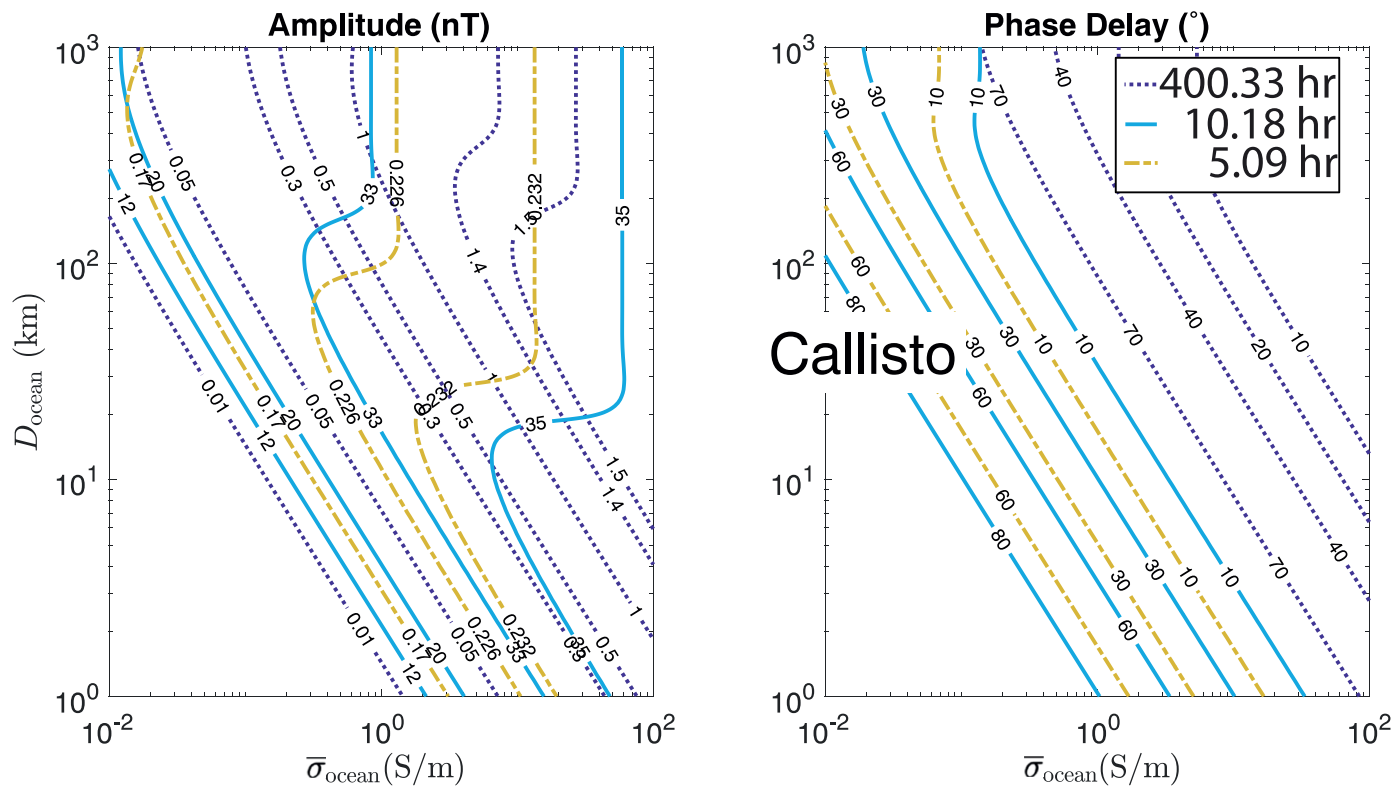

Figure 4. Callisto: Contours of the maximum induced field $B_{y}$ components (in nT) and phase delays (in ${ }^{\circ}$ ) at the strongest inducing periods—orbital (400.33 h; dotted), Jupiter synodic (10.18 h; solid), and second synodic harmonic ( 5.09 h; dot-dash)—shown in Figure 1. Additional harmonic short-period components will be advantageous for investigating Callisto's interior structure. The normalized amplitudes and phases for the synodic frequencies are consistent with those described by Zimmer et al. (2000). The amplitudes and phases for the synodic and orbital periods are similar to those described by Seufert et al. (2011), but these authors model a moderately conducting silicate interior, which we do not consider. A $50 \mathrm{~km}$ ice shell is assumed at the surface, implying that the seafloor depth varies to accommodate the range of $D_{\text {ocean }}$. 
locations. For example, the largest variation at Ganymede's synodic period is in its $B_{y}$ component in $G \phi \Omega$ coordinates, approximately along the direction toward Jupiter. If a lander at the sub or antijovian point on Ganymede's surface measures an induced field amplitude of $75 \mathrm{nT}$ at that period, the matching ocean thickness $D_{\text {ocean }}$ and mean conductivity $\sigma_{\text {ocean }}$ must lie along the $75 \mathrm{nT}$ contour. Ganymede's orbital period also has its largest oscillation in $B_{y}$, so including the measured amplitude at that period too determines the values for both $D_{\text {ocean }}$ and $\sigma_{\text {ocean }}$, at the crossover point between the two contours. The phase delay for each frequency offers complementary information.

In contrast with the parameter exploration reproduced here and employed in previous work, we allow ice thickness to vary. We consider how the ocean conductivity varies in accordance with the ice thickness: the melting temperature at the base sets the adiabatic temperature of the ocean, and is determined by the ocean's salinity and the pressure at the base of the ice (Vance et al., 2018). Also in contrast with the parameter space exploration depicted in Figures 2-4, we examine a smaller space of $\sigma_{\text {ocean }}$ and $D_{\text {ocean }}$ consistent with previous models of Europa's ocean composition, as described in the next section and summarized in Tables 2-4.

In this work, we do not consider the effect on the diffusive induction signal from a possible highly conductive metallic core or moderately conductive, hydrated rocky mantle in any of the satellites. One past study of Europa by Schilling (2006) determined that for even modest ocean conductivities ( $\gtrsim 0.06 \mathrm{~S} / \mathrm{m})$, the presence of a core would be all but undetectable. A mantle would similarly be easily screened by a moderately conductive ocean. Seufert et al. (2011), however, found that for some combinations of $D_{\text {ocean }}$ and $\sigma_{\text {ocean }}$, a metallic core would change the amplitude of the diffusive response by several percent and decrease the phase delay by $10^{\circ}$ or more. A conductive core will have the most dramatic effect for the thinnest and least conductive ocean layers, at the bottom-left of Figures 2-4. For an ocean that fails to entirely screen a highly conductive core, new contours with a smaller phase delay appear in this corner of the plot. Modeling the wide parameter space of possible interior configurations that also include a core or mantle is beyond the scope of this work.

We also add to the rich set of previous analyses the exploration of a third, shorter-period signal of intermediate strength to the orbital and synodic signals. We do not consider the longer-period solar oscillation studied by Seufert et al. (2011).

\subsection{Depth-Dependent Electrical Conductivity in Adiabatic Oceans}

Here we describe the structural models that allow us to consider depth-dependent electrical conductivity in Jupiter's ocean moons. Fluid temperature, pressure, and salt content determine the electrical conductivity of an aqueous solution, and thus dictate the magnetic induction responses of the Galilean oceans. With sufficient prior knowledge of the ice thickness and the ocean's composition-for example, from geological and compositional measurements by the planned Europa Clipper mission (Buffington et al., 2017)—magnetic induction studies can provide information on the amounts and compositions of the salts that link to global thermal and geochemical processes.

Depth-dependence in the ocean's electrical conductivity can arise from stratification in the ocean due to melting or freezing at the ice-ocean interface, and dissolution and precipitation within the ocean or at the water-rock interface (Travis et al, 2012; Vance \& Brown, 2005). Even for oceans with uniform salinity, as is typically assumed, electrical conductivity will increase with depth along the ocean's convective adiabatic profile because the greater temperature and pressure increase the electrical conductivity. Figure 5 depicts this variation for Europa, Ganymede, and Callisto, based on the forward models of Vance et al. (2018) that use available thermodynamic and geophysical data to explore the influences of the ocean, rock layer, and any metallic core on the radial structures of known icy ocean worlds. As noted by Hand and Chyba (2007), the adiabatic gradient for Europa is rather small, albeit nonzero. A more significant influence on the ocean's temperature is the influence of pressure on the melting temperature of the ice, which in turn depends on the ocean's salinity. For Ganymede and Callisto, the adiabatic gradients are large, with temperatures at the base of the thickest Ganymede ocean reaching 290K.

As detailed in Section 2.2, we examine the magnetic induction signals from the small set of self-consistent adiabatic ocean models, taken primarily from those described in detail by Vance et al. (2018). Minor 
Table 2

Europa: Magnetic Induction Field Strengths $\{\operatorname{Re}, \operatorname{Im}\}\left(B_{y} \mathcal{A}_{1}^{e}\right)$, in $n T$, at the Main Inducing Periods in Figure 1

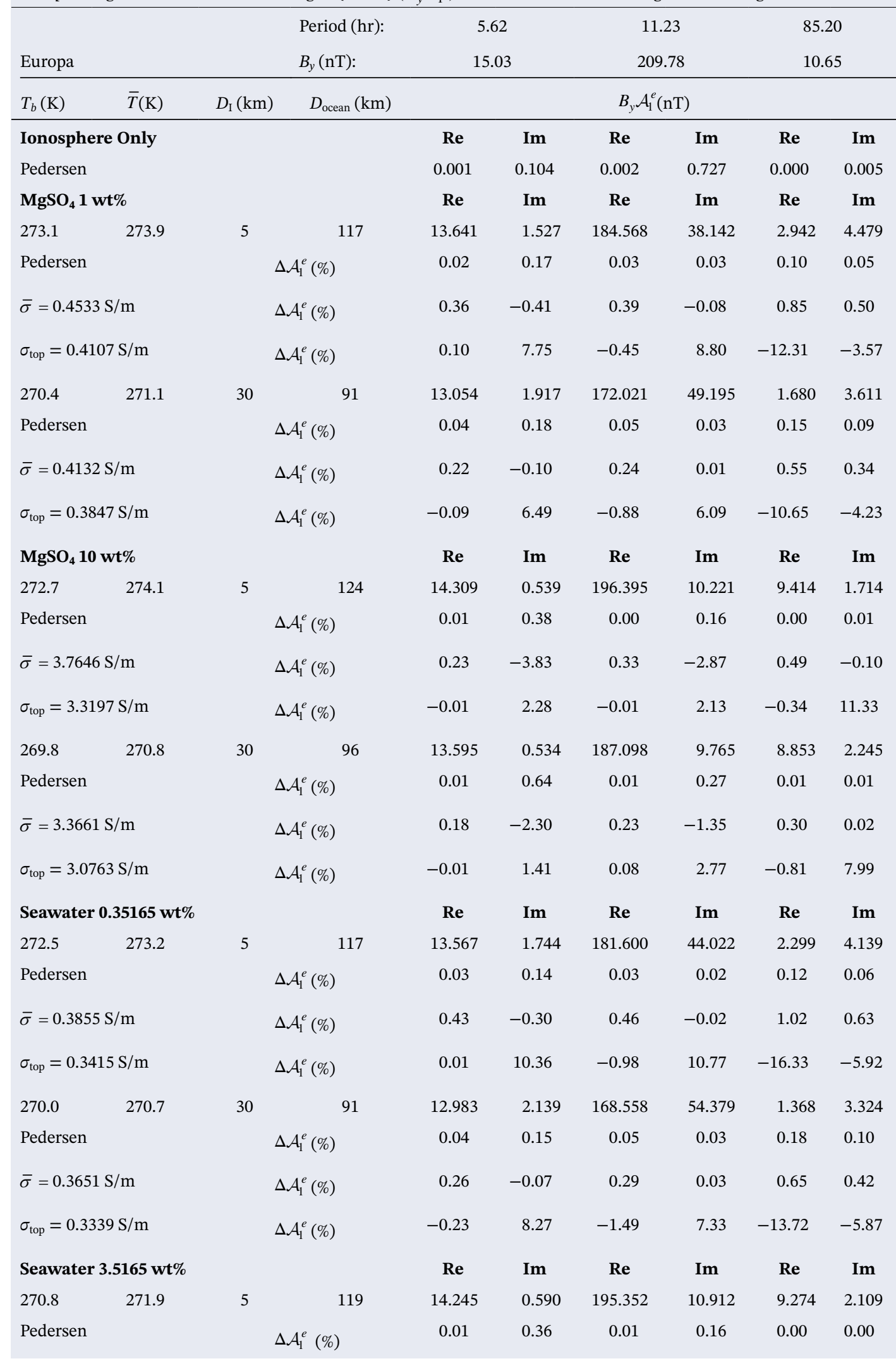




\begin{tabular}{|c|c|c|c|c|c|c|c|c|}
\hline \multirow[b]{2}{*}{ Europa } & \multirow{2}{*}{\multicolumn{2}{|c|}{$\begin{array}{l}\text { Period }(\mathrm{hr}) \text { : } \\
B_{y}(\mathrm{nT}):\end{array}$}} & \multicolumn{2}{|c|}{5.62} & \multicolumn{2}{|c|}{11.23} & \multicolumn{2}{|c|}{85.20} \\
\hline & & & \multicolumn{2}{|c|}{15.03} & \multicolumn{2}{|c|}{209.78} & \multicolumn{2}{|c|}{10.65} \\
\hline$T_{b}(\mathrm{~K})$ & $D_{\mathrm{I}}(\mathrm{km})$ & $D_{\text {ocean }}(\mathrm{km})$ & \multicolumn{6}{|c|}{$B_{y} \mathcal{A}_{1}^{e}(\mathrm{nT})$} \\
\hline $\bar{\sigma}=3.0760 \mathrm{~S} / \mathrm{m}$ & & $4_{1}^{e}(\%)$ & 0.24 & -3.32 & 0.33 & -2.24 & 0.46 & -0.03 \\
\hline$\sigma_{\text {top }}=2.7347 \mathrm{~S} / \mathrm{m}$ & & $4_{1}^{e}(\%)$ & -0.02 & 2.08 & 0.04 & 2.37 & -0.74 & 10.53 \\
\hline 269.1 & 30 & 91 & 13.530 & 0.560 & 186.582 & 10.460 & 8.612 & 2.664 \\
\hline Pedersen & & $f_{1}^{e}(\%)$ & 0.01 & 0.63 & 0.01 & 0.26 & 0.01 & 0.00 \\
\hline $\bar{\sigma}=2.8862 \mathrm{~S} / \mathrm{m}$ & & $f_{1}^{e}(\%)$ & 0.18 & -1.89 & 0.22 & -0.95 & 0.28 & 0.03 \\
\hline$\sigma_{\mathrm{top}}=2.6476 \mathrm{~S} / \mathrm{m}$ & $\Delta$ & $A_{1}^{e}(\%)$ & 0.01 & 1.46 & 0.10 & 3.88 & -1.26 & 7.23 \\
\hline
\end{tabular}

Note. For the different ocean compositions and thicknesses of the upper ice I lithosphere/ocean $\left(\mathrm{D}_{\mathrm{I}} / \mathrm{D}_{\text {ocean }}\right.$; Figure 5$)$, the adiabatic response is listed first. These values are also shown in Figure 7. Following these are the deviations from the adiabatic response (in \%) when including a $100 \mathrm{~km}$ ionosphere with Pedersen conductance of $30 \mathrm{~S}$ (Hartkorn \& Saur, 2017), then for the ocean with uniform conductivity set to the mean of the adiabatic ocean $(\bar{\sigma})$, and then for the case with uniform conductivity set to the value at the ice-ocean interface $\left(\sigma_{\text {top }}\right)$. The surface responses of the ionosphere in the absence of an ocean are listed at the top of the table.

changes to the PlanetProfile software used to generate the models (Melwani Daswani et al., under revision, S3) do not significantly change the ocean thicknesses and electrical conductivities reported in the previous work. We do not consider significant induction from rocky or metallic layers. For each ocean, we consider a nominal $10 \mathrm{wt} \% \mathrm{MgSO}_{4}$ salinity, as investigated in previous work. The published equation of state and electrical conductivity data are adequate for the pressures in the largest moon, Ganymede, up to $1.6 \mathrm{GPa}$, with the caveat that both have been extrapolated in pressure above about $0.7 \mathrm{GPa}$, and the laboratory data for electrical conductivity have been extrapolated below $298 \mathrm{~K}$ and above $1 \mathrm{wt} \%$ (Vance et al., 2018). The pressure conditions in Europa's ocean are low enough ( $<200 \mathrm{MPa})$ to be in the range covered by the TEOS10 package (McDougall \& Barker, 2011), which provides plausible values of conductivity for concentrations of seawater equivalent to that of Earth's ocean $(3.5 \mathrm{wt} \% \mathrm{NaCl}$ ) or less. For this work, we created additional lower-conductivity models for the same ice thickness, but with salinities reduced by a factor of 10 from the nominal cases.

On Europa, the flux of surface-generated oxygen to the ocean may have created oxidizing (acidic) conditions (Hand \& Chyba, 2007; Pasek \& Greenberg, 2012; Vance et al., 2016), permitting the presence of dissolved $\mathrm{MgSO}_{4}$ in addition to $\mathrm{NaCl}$ (Zolotov, 2008; Zolotov \& Kargel, 2009). The respective radial models of electrical conductivity for oceans containing seawater and $\mathrm{MgSO}_{4}$ are consistent with compositions linked to the thermal evolution scenarios cited above (Zolotov \& Kargel, 2009). In one scenario, Europa's ocean remains relatively reducing and high $\mathrm{pH}$, with a composition dominated by $\mathrm{NaCl}$. In the other, the flux into the ocean of oxidants generated by radiolysis of Europa's ice causes the ocean to become more oxidized and low $\mathrm{pH}$, containing quantities of $\mathrm{MgSO}_{4}$ exceeding the amount of $\mathrm{NaCl}$. Thus the ocean's salinity and composition that might be constrained by magnetic induction measurements relate to the thermal history of Europa. The salinity measurement is also a key indicator of the types of life that might be able to live in the ocean because the chemical affinity-or energy in excess of equilibrium-for different metabolic reactions depends on the ocean's pH (Glein et al., 2019).

Radial conductivity profiles for Europa (Figure 5; top) illustrate the coupling to temperature and composition. We consider ice thicknesses of 5 and $30 \mathrm{~km}$ (magenta and blue curves, respectively) as representative extremes. Because we consider only the mean inferred value of the gravitational moment of inertia $(C /$ $M R^{2}=0.346 \pm 0.005$ Schubert et al., 2004), the hydrosphere thickness is fixed at about $125 \mathrm{~km}$. Seawater (solid and dot-dashed lines), though less concentrated than the modeled composition of $\mathrm{MgSO}_{4}$ (dashed lines), has a stronger melting point suppression, leading to an overall colder ocean for the same thickness of 
Table 3

Ganymede: Magnetic Induction Field Strengths $\{\operatorname{Re}, \operatorname{Im}\}\left(B_{y} \mathcal{A}_{1}^{e}\right)$, in $n T$, at the Main Inducing Periods in 1

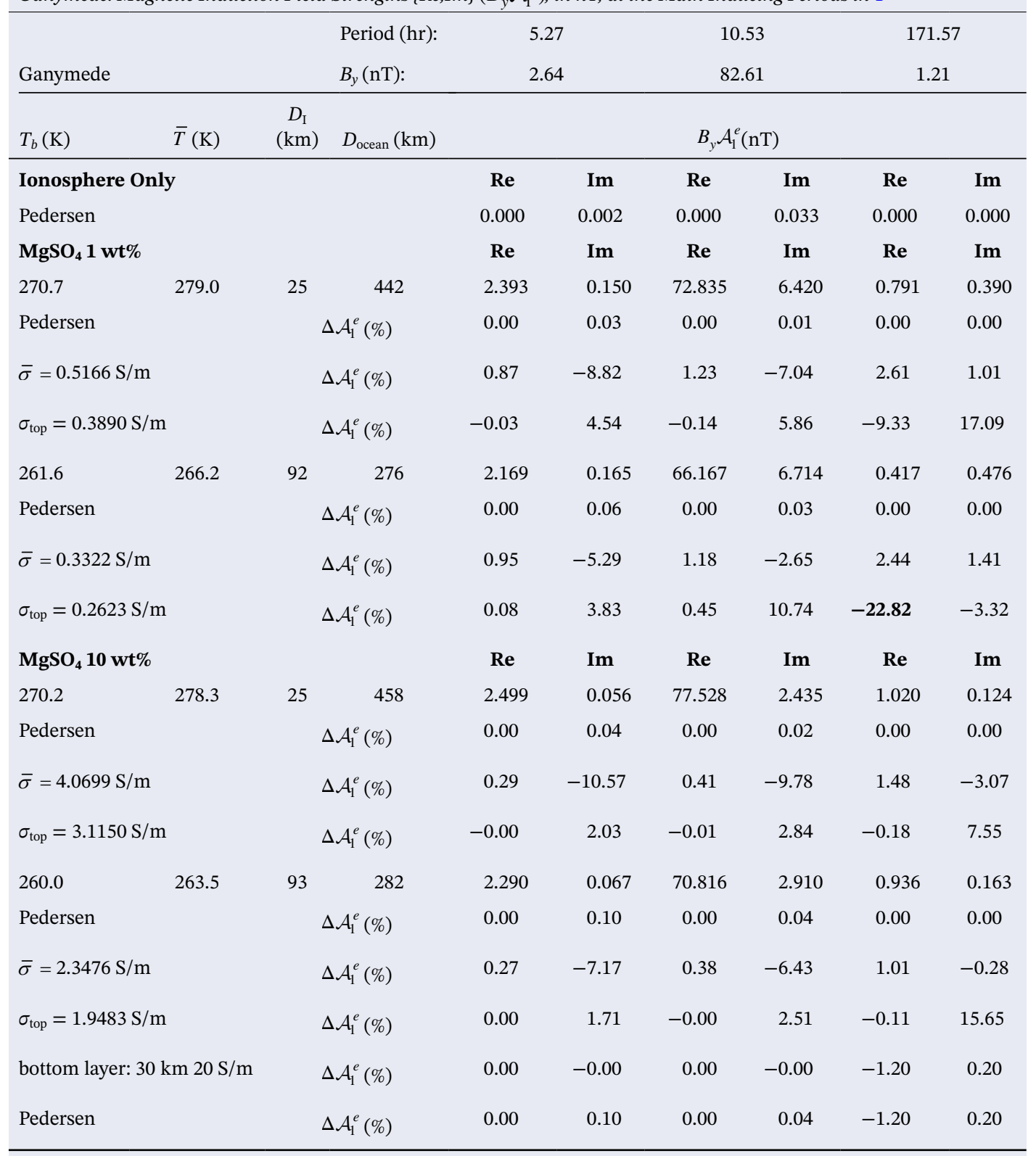

For the different ocean compositions and thicknesses of the upper ice I lithosphere (DI; Figure 5), the adiabatic response is listed first. These values are also shown in Figure 7. Following these are deviations from the adiabatic response (in \%) when including a $100 \mathrm{~km}$ ionosphere with Pedersen conductance of $2 \mathrm{~S}$ (Hartkorn \& Saur, 2017), then for the ocean with uniform conductivity set to the mean of the adiabatic ocean $(\bar{\sigma})$, and then for the case with uniform conductivity set to the value at the ice-ocean interface ( $\sigma$ top). The surface responses of the ionosphere in the absence of an ocean are listed at the top of the table.

ice. The lower temperature for seawater combines with the different electrical conductivity for the different dissolved ions to create distinct profiles unique to ocean composition and ice thickness (upper right). As a result, our conductivity values differ from the summary predictions in Figure 1 of Hand and Chyba (2007) for $T=0^{\circ} \mathrm{C}$ and $1 \mathrm{~atm}$. This discrepancy from previously published values of electrical conductivity is further evident in the larger moons Ganymede and Callisto, where ocean temperatures vary farther from the freezing point at standard temperature and pressure. 
Table 4

Callisto: Magnetic Induction Field Strengths $\{\operatorname{Re}, \operatorname{Im}\}\left(B_{y} \mathcal{A}_{1}^{e}\right)$, in $n T$, at the Main Inducing Periods in Figure 1

\begin{tabular}{|c|c|c|c|c|c|c|c|c|c|}
\hline \multirow{2}{*}{\multicolumn{2}{|c|}{ Callisto }} & \multirow{2}{*}{\multicolumn{2}{|c|}{$\begin{array}{l}\text { Period (hr): } \\
B_{y}(\mathrm{nT}):\end{array}$}} & \multirow{2}{*}{\multicolumn{2}{|c|}{$\begin{array}{l}5.09 \\
0.25\end{array}$}} & \multirow{2}{*}{\multicolumn{2}{|c|}{$\begin{array}{l}10.18 \\
37.57\end{array}$}} & \multirow{2}{*}{\multicolumn{2}{|c|}{$\begin{array}{c}400.33 \\
1.72\end{array}$}} \\
\hline & & & & & & & & & \\
\hline$T_{b}(\mathrm{~K})$ & \multirow[t]{2}{*}{$\bar{T}(\mathrm{~K})$} & \multirow[t]{2}{*}{$D_{\mathrm{I}}(\mathrm{km})$} & \multirow[t]{2}{*}{$D_{\text {ocean }}(\mathrm{km})$} & \multicolumn{6}{|c|}{$B_{y} \mathcal{A}_{1}^{e}(\mathrm{nT})$} \\
\hline Ionosphere Only & & & & $\mathbf{R e}$ & Im & $\mathbf{R e}$ & Im & $\mathbf{R e}$ & Im \\
\hline \multicolumn{2}{|c|}{ Pedersen } & & & 0.019 & 0.070 & 0.769 & 5.549 & 0.000 & 0.007 \\
\hline \multicolumn{2}{|c|}{ Cowling } & & & 0.230 & 0.097 & 23.854 & 20.120 & 0.002 & 0.056 \\
\hline \multicolumn{2}{|c|}{$\mathrm{MgSO}_{4} 1 \mathrm{wt} \%$} & & & $\mathbf{R e}$ & Im & $\operatorname{Re}$ & Im & $\operatorname{Re}$ & Im \\
\hline 257.4 & 259.6 & 99 & 132 & 0.204 & 0.023 & 29.774 & 6.332 & 0.021 & 0.171 \\
\hline \multicolumn{2}{|c|}{ Pedersen } & & & 0.207 & 0.026 & 30.227 & 6.544 & 0.022 & 0.177 \\
\hline \multicolumn{2}{|c|}{ Cowling } & & & 0.231 & 0.036 & 33.248 & 7.167 & 0.033 & 0.225 \\
\hline \multicolumn{2}{|c|}{$\bar{\sigma}=0.2307 \mathrm{~S} / \mathrm{m}$} & & $\Delta \mathcal{A}_{1}^{e}(\%)$ & 0.49 & -0.44 & 0.53 & -0.08 & 1.45 & 0.96 \\
\hline \multicolumn{2}{|c|}{$\sigma_{\mathrm{top}}=0.1965 \mathrm{~S} / \mathrm{m}$} & & $\Delta \mathcal{A}_{1}^{e}(\%)$ & 0.06 & 14.62 & -1.03 & 15.03 & -26.08 & -13.62 \\
\hline 250.8 & 250.9 & 128 & 21 & 0.060 & 0.095 & 2.885 & 9.085 & 0.000 & 0.012 \\
\hline \multicolumn{2}{|c|}{ Pedersen } & & & 0.102 & 0.119 & 5.702 & 13.168 & 0.000 & 0.018 \\
\hline \multicolumn{2}{|c|}{ Cowling } & & & 0.238 & 0.083 & 27.259 & 18.811 & 0.003 & 0.068 \\
\hline \multicolumn{2}{|c|}{$\bar{\sigma}=0.0895 \mathrm{~S} / \mathrm{m}$} & & $\Delta \mathcal{A}_{1}^{e}(\%)$ & 0.04 & 0.02 & 0.04 & 0.03 & 0.05 & 0.03 \\
\hline \multicolumn{2}{|c|}{$\sigma_{\mathrm{top}}=0.0874 \mathrm{~S} / \mathrm{m}$} & & $\Delta \mathcal{A}_{1}^{e}(\%)$ & -3.26 & -0.99 & -4.12 & -1.87 & -4.52 & -2.28 \\
\hline \multicolumn{2}{|c|}{$\mathrm{MgSO}_{4} 10 \mathrm{wt} \%$} & & & $\mathbf{R e}$ & Im & $\mathbf{R e}$ & Im & $\mathbf{R e}$ & Im \\
\hline 255.7 & 256.9 & 99 & 130 & 0.211 & 0.008 & 31.391 & 1.533 & 0.552 & 0.696 \\
\hline \multicolumn{2}{|c|}{ Pedersen } & & & 0.212 & 0.011 & 31.490 & 1.787 & 0.556 & 0.698 \\
\hline \multicolumn{2}{|c|}{ Cowling } & & & 0.226 & 0.027 & 32.566 & 3.378 & 0.582 & 0.715 \\
\hline \multicolumn{2}{|c|}{$\bar{\sigma}=1.5256 \mathrm{~S} / \mathrm{m}$} & & $\Delta \mathcal{A}_{1}^{e}(\%)$ & 0.20 & -2.91 & 0.26 & -1.74 & 0.69 & 0.39 \\
\hline \multicolumn{2}{|c|}{$\sigma_{\text {top }}=1.3789 \mathrm{~S} / \mathrm{m}$} & & $\Delta \mathcal{A}_{1}^{e}(\%)$ & 0.01 & 1.12 & 0.12 & 3.18 & -10.78 & -1.59 \\
\hline 250.8 & 250.9 & 128 & 21 & 0.195 & 0.053 & 24.308 & 13.231 & 0.003 & 0.067 \\
\hline Peders & & & & 0.202 & 0.055 & 25.716 & 13.402 & 0.004 & 0.074 \\
\hline Cowli & & & & 0.239 & 0.049 & 32.873 & 12.030 & 0.009 & 0.123 \\
\hline $\bar{\sigma}=0$. & & & $\Delta \mathcal{A}_{1}^{e}(\%)$ & -0.00 & -0.00 & -0.00 & -0.00 & -0.00 & -0.00 \\
\hline$\sigma_{\text {top }}=$ & $\mathrm{S} / \mathrm{m}$ & & $\Delta \mathcal{A}_{1}^{e}(\%)$ & 0.08 & -0.53 & 0.28 & -0.34 & 1.23 & 0.61 \\
\hline
\end{tabular}

Note. For the different ocean compositions and thicknesses of the upper ice I lithosphere/ocean $\left(\mathrm{D}_{\mathrm{I}} / \mathrm{D}_{\text {ocean }}\right.$; Figure 5$)$, the adiabatic response is listed first. These values are also shown in Figure 7. Following these are the responses (in $\mathrm{nT}$ ) including a $100 \mathrm{~km}$ ionosphere with \{Pedersen, Cowling\} conductance of $\{800,6850\}$ S (Hartkorn \& Saur, 2017), then the deviations from the adiabatic response (in \%) for the ocean with uniform conductivity set to the mean of the adiabatic ocean $(\bar{\sigma})$, and then for the case with uniform conductivity set to the value at the ice-ocean interface $\left(\sigma_{\text {top }}\right)$. The surface responses of the ionosphere in the absence of an ocean are listed at the top of the table.

Although we also fix the moments of inertia for Ganymede and Callisto to their mean published values, the depths of the ocean vary due to the presence of high-pressure ices (as further discussed in Section S3). Because the melting of high-pressure ices also depends on pressure (e.g., Hogenboom et al., 1995) the presence of ices above and below the ocean increases the sensitivity of the ocean's conductance to the composition and abundance of dissolved salts.

Larger Ganymede (Figure 5; middle) has distinct conductivity profiles for both ice thickness and ocean composition. Although electrical conductivity generally increases with depth, it begins to decrease at the 

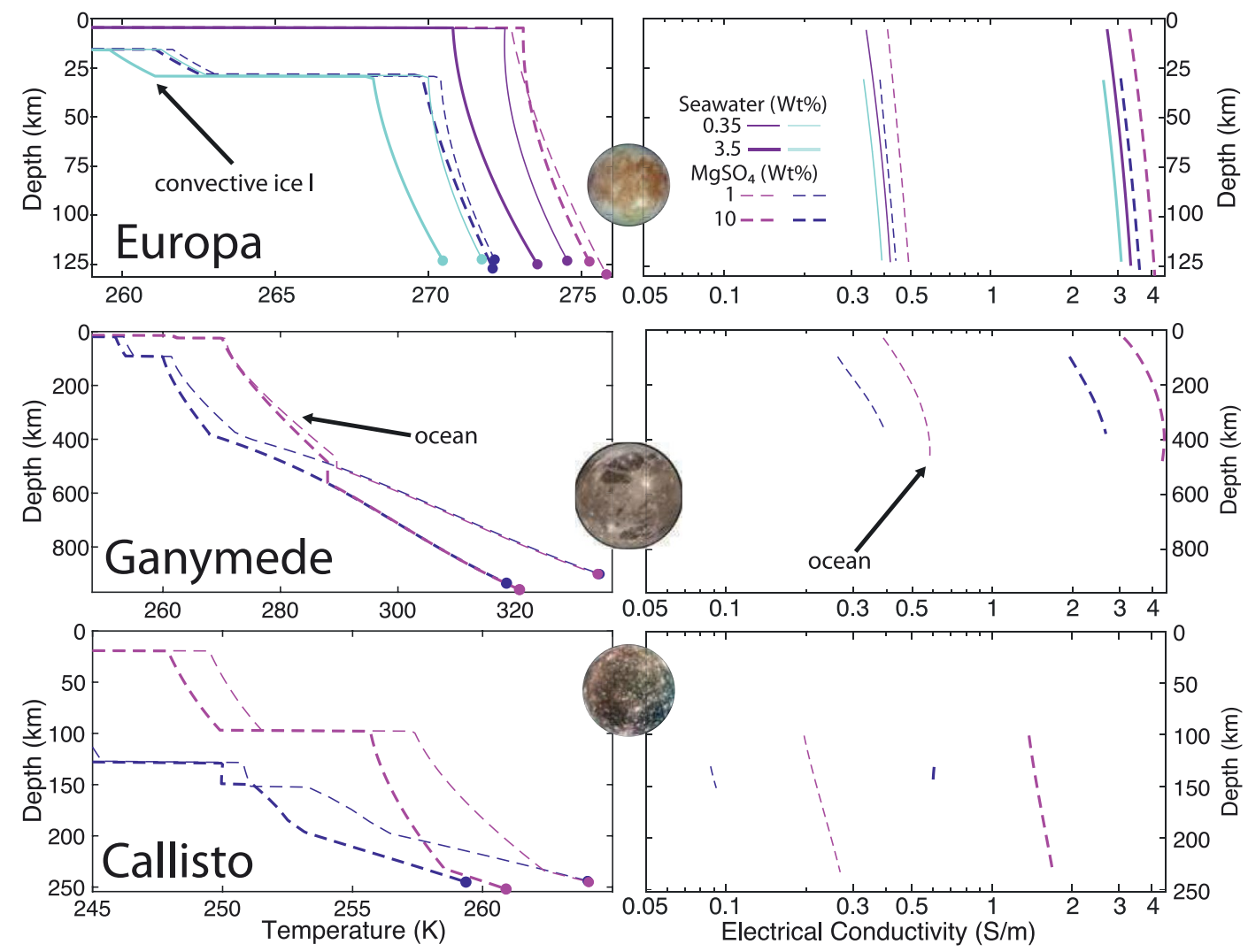

Figure 5. Adiabatic ocean temperature (left) and electrical conductivity (right). Convecting oceans with $\mathrm{MgSO}_{4}$ (dashed lines) are warmer. Standard seawater (mostly NaCl; dot-dashed lines) creates colder oceans and lower electrical conductivities. Thicker ice (blue), corresponds to colder adiabatic profiles in the underlying oceans, which also lowers electrical conductivity. Filled circles show the inferred depth to the upper boundary of the silicate layer for the saline and pure water oceans, respectively. Conductivities in the liquid regions are several orders of magnitude larger than in the ice and rock, and are set to zero for this study. Adapted from Vance et al. (2018).

greatest depths for the warm Ganymede ocean (right-most curve). This inflection occurs because the ocean achieves $\mathrm{GPa}+$ pressures, at which the packing of water molecules begins to inhibit the charge exchange of the dissolved ions (Schmidt \& Manning, 2017).

Dense brines may also reside at the base of the high-pressure ices on Ganymede, and even between them (Journaux et al., 2013, 2017; Vance et al., 2014, 2018). Although more detailed modeling of the coupled geochemical and geodynamic regimes is needed, this scenario seems consistent with recent simulations of two-phase convection in high-pressure ices (Choblet et al., 2017; Kalousová et al., 2018). These simulations show that even without the effects of dissolved salts, meltwater should form at the water-rock interface as part of the geodynamic evolution of the ice. If such a stable fluid layer exists under the high-pressure ice within Ganymede, it will create an induction response at longer periods, as discussed below.

For Callisto, there is a small range of ice I thicknesses and ocean salinities for which oceans may be present. Salty oceans considered by Vance et al. (2018) have thicknesses of 20 and $132 \mathrm{~km}$. For the thinner ocean, a $96 \mathrm{~km}$ layer of high-pressure ice underlies the ocean. The depicted state is likely transient, as ice III is buoyant in the modeled $10 \mathrm{wt} \% \mathrm{MgSO}_{4}$ composition, and an upward snow effect should hasten the transfer of heat from the interior. Simulating a subsequent stage with ice III above the ocean awaits improved thermodynamic data that couples recently improved ice thermodynamics (Journaux et al., 2020) to the thermodynamics of aqueous phases (Bollengier et al., 2019), and is left for future work. Because of the thicker ice considered for Callisto and the consequentially lower temperature at the upper ice-ocean interface, the electrical conductivities in all Callisto models are lower than for the corresponding concentrations in Ganymede. In terms of the magnetic induction response, as shown in Section 2.6, these lower conductivity values 


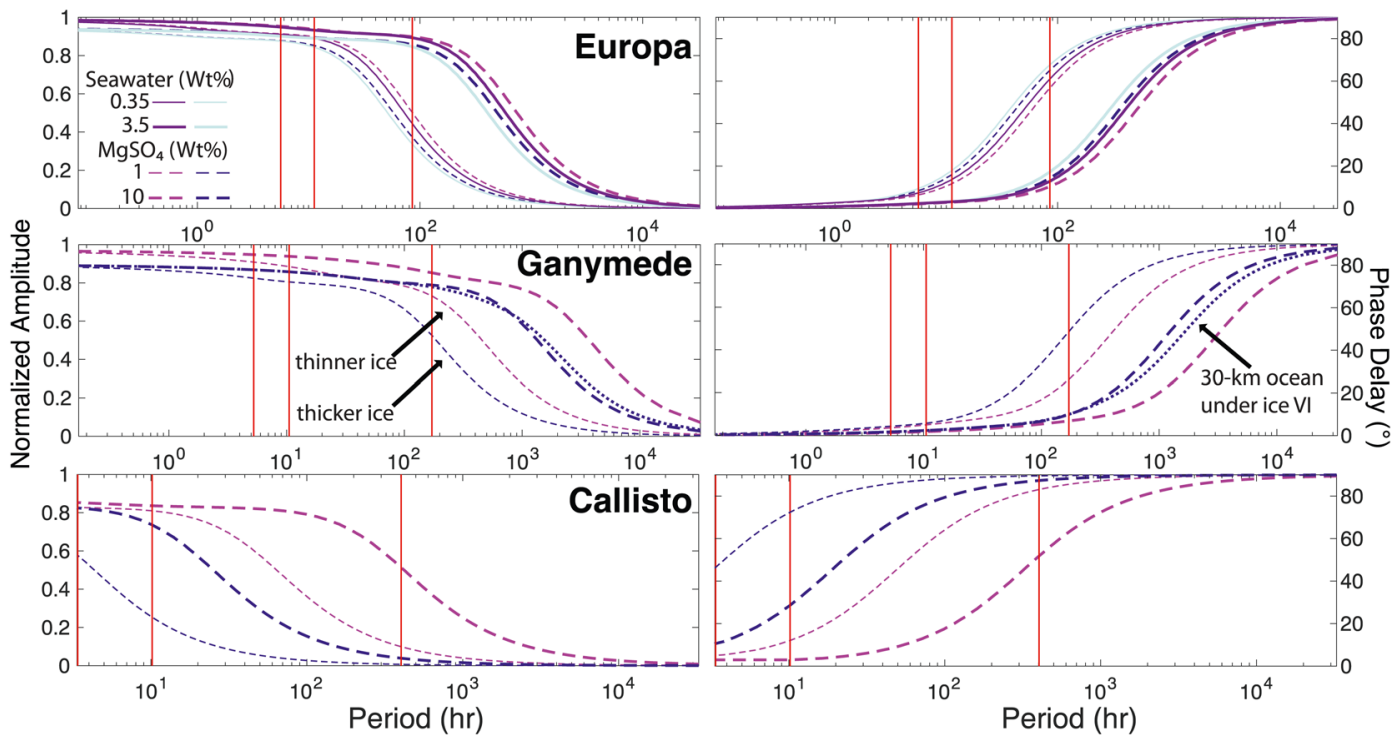

Figure 6. Normalized magnetic induction amplitudes $\left(A=\left|\mathcal{A}_{1}^{e} \quad\right|\right.$; left $)$ and phase delays $\left(\phi=-\arg \left(\mathcal{A}_{1}^{e}\right)\right.$; right $)$ for Europa, Ganymede, and Callisto at periods including the induction peaks noted in Figure 1 (vertical red lines). As in Figure 5, dashed lines are for oceans containing $\mathrm{MgSO}_{4}$. Solid and dot-dashed lines are for oceans containing seawater (mostly $\mathrm{NaCl}$ ). Thicker lines have higher concentrations of $\{10,3.5\} \mathrm{wt} \%$, respectively, and thinner lines correspond to oceans diluted by a factor of 10 . For the $\mathrm{MgSO}_{4}$-bearing oceans, thinner ice corresponding to warmer oceans is denoted with magenta and thicker ice is dark blue. The trends with ice thickness/ocean temperature are the same for seawater oceans: larger amplitude and lower phase delay for thinner ice/warmer oceans. For Ganymede, the dotted line indicates the effect of introducing a 30-km-thick, $20 \mathrm{~S} / \mathrm{m}$ layer at the seafloor for the thick-ice and high-salinity ocean, which is the thicker blue dashed line.

compound the lower overall conductance resulting from the thinner ocean, and also the smaller driving magnetic oscillations at more distant Callisto.

\subsection{Accounting for the Ionospheres}

For each of the above models, we add an overlying ionospheric layer based on recent analyses by Hartkorn and Saur (2017). We adopt their simplified ionospheric models, while also noting that the detailed radial and asymmetric structures of the ionospheres will affect the complex induction response and should be considered in future work. For each satellite, we consider a 100-km-thick layer extending from the surface, with Pedersen conductances of $\{30,2,800\}$ S for Europa, Ganymede, and Callisto, respectively. For Callisto, we also consider a higher value of $6850 \mathrm{~S}$ corresponding to a Cowling channel enhancement near the equator arising from anisotropy in the current sheet, consistent with Hartkorn and Saur (2017). We use this value as an extreme case to inform the analysis of measurements near the equator. In reality, the nonspherical character of the ionosphere will influence the induction response from the one computed here, perhaps up to the order of nT (Styczinski \& Harnett, 2021). The enhancement of the Cowling effect is expected to create an effective conductance only twice that of the Pedersen value at higher latitudes. For clarity in presenting the results, the effects of the ionosphere are included only in the tabulated results (Tables 2-4). Amplitudes are normalized to the moons' surface radii $R$ : $A_{\text {surf }}=\left(R_{\text {top }} / R\right)^{3} A$, where $R_{\text {top }}=R+100 \mathrm{~km}$, so they can be larger than unity.

\subsection{Amplitude and Phase Delay of the Diffusive Response}

Figure 6 shows the normalized surface induction responses for Europa, Ganymede, and Callisto based on the adiabatic ocean electrical conductivity profiles shown in Figure 5. Some general characteristics of the induction response may be discerned. Warmer and thus thicker oceans (magenta curves for $\mathrm{MgSO}_{4} \mathrm{Com}_{-}$ positions) have larger amplitude responses, corresponding to overall higher values of the conductance. For 
longer periods, the influence of salinity on the amplitude responses dominate, while the thickness of the ocean dominates at shorter periods. Amplitudes approach zero around periods of $10^{4} \mathrm{~h}$. Less saline oceans have more significant phase delays at longer periods.

For Europa, the induction characteristics for modeled oxidized (10 wt $\mathrm{MgSO}_{4}$ ) and reduced (seawater) oceans are nearly identical in their amplitude responses. However, the two ocean models show a separation in phase delay of a few degrees at the orbital period of $85.20 \mathrm{~h}$. The combination of these features that constitutes the complex induction waveform will be key to separating them, as shown in Section 2.6.

Regional enhancements in the ocean conductivity can have a significant induction response. For Ganymede, we simulate a second ocean layer at the water-rock interface at a depth of $900 \mathrm{~km}$. Lying under $530 \mathrm{~km}$ of ice VI (Vance et al., 2018), this layer is modeled as a 30-km-thick high-conductivity region (20 S/m) corresponding to a nearly saturated $\mathrm{MgSO}_{4}$ solution, consistent with (Hogenboom et al., 1995) and (Calvert et al., 1958). The influence of such a layer (dotted lines in Figure 6) is a 1\% decrease in amplitude at the orbital period of $171.57 \mathrm{~h}$. The amplitude decrease results from mutual induction between the conducting layers at this period.

For Callisto, the present simulations illustrate the influence of the thicker and deeper oceans in terms of a higher amplitude response at lower frequencies and a phase delay curve also shifted in the direction of lower frequencies.

\subsection{Distinguishing Diffusive Responses for Different Model Oceans}

We examine the possible separability of different model oceans by plotting the real and imaginary components of the induced waveforms for the peak values of Jupiter's inducing field vectors. Figure 7 shows the real and imaginary parts of the complex diffusive induction response. The normalized complex response $\mathcal{A}_{n}^{e}$ is multiplied by the strength of the excitation field $B_{y}$ at the driving periods shown in Figure 1 , in accordance with Equation 8. $\mathcal{A}_{1}^{e}$ is equal to $A e^{-i \phi}$, with the normalized amplitude $A$ and phase delay $\phi$ equal to those used in past studies such as Zimmer et al. (2000; see Section S1). Previous authors (including Zimmer et al., 2000) have defined the complex response as $A e^{i \phi}$, but they obtain a result equal to the complex conjugate of $\mathcal{A}_{1}^{e}$ because they rely on a derivation in Parkinson (1983) that contains an error (see Section S1). Relating $\mathcal{A}_{1}^{e}$ to $A$ and $\phi$ as we do enables us to use the same representation as past authors in comparing the induced magnetic field to that which would result from a perfectly conducting ocean $\mathbf{B}_{\mathrm{dif}, \infty}$ at an earlier time $t-\phi / \omega$ :

$$
\mathbf{B}_{\text {dif }}(t)=A \mathbf{B}_{\text {dif }, \infty}(t-\phi / \omega)
$$

If we were to instead define $\mathcal{A}_{1}^{e}$ as equal to $A e^{i \phi_{\mathrm{conj}}},-90^{\circ} \leq \phi_{\mathrm{conj}}<0^{\circ}$ and Equation 10 would then become

$$
\mathbf{B}_{\mathrm{dif}}(t)=A \mathbf{B}_{\mathrm{dif}, \infty}\left(t+\phi_{\text {conj }} / \omega\right)
$$

Both definitions represent the same physical result.

The quantities $B_{y} \mid\{\operatorname{Re}, \operatorname{Im}\}\left(\mathcal{A}_{1}^{e}\right)$ I, equivalent to $B_{y} A \cos \phi$ and $B_{y} A \sin \phi$, describe the strengths of the responses that are in phase with the excitation field-an instantaneous response that opposes the external field-and the component that is exactly $90^{\circ}$ out-of-phase, respectively. Thus, the two components together describe the full range of the induction response. Tables 2-4 include the corresponding data; absolute values are implied on the out-of-phase components, consistent with considering spectral information and required by the choice of positive phase delay as in Equation 10. These tables also provide the computed values that include the modeled ionospheres, and the values computed for the equivalent oceans with the conductivity set to the mean of the adiabat and to the value at the top of the ocean. For convenience, Figures S6 and S7 and Tables S1-S6 provide the corresponding data for $B_{x}$ and $B_{z}$; these corresponding values may also be obtained by substituting the field strengths in Table 1 in the data and tables for $B_{y}$.

\subsubsection{Europa}

The different phase delays and amplitudes at the orbital and synodic harmonic periods described in Section 2.5 create differences in the induction responses for different models of as much as $25 \mathrm{nT}$, comparing 

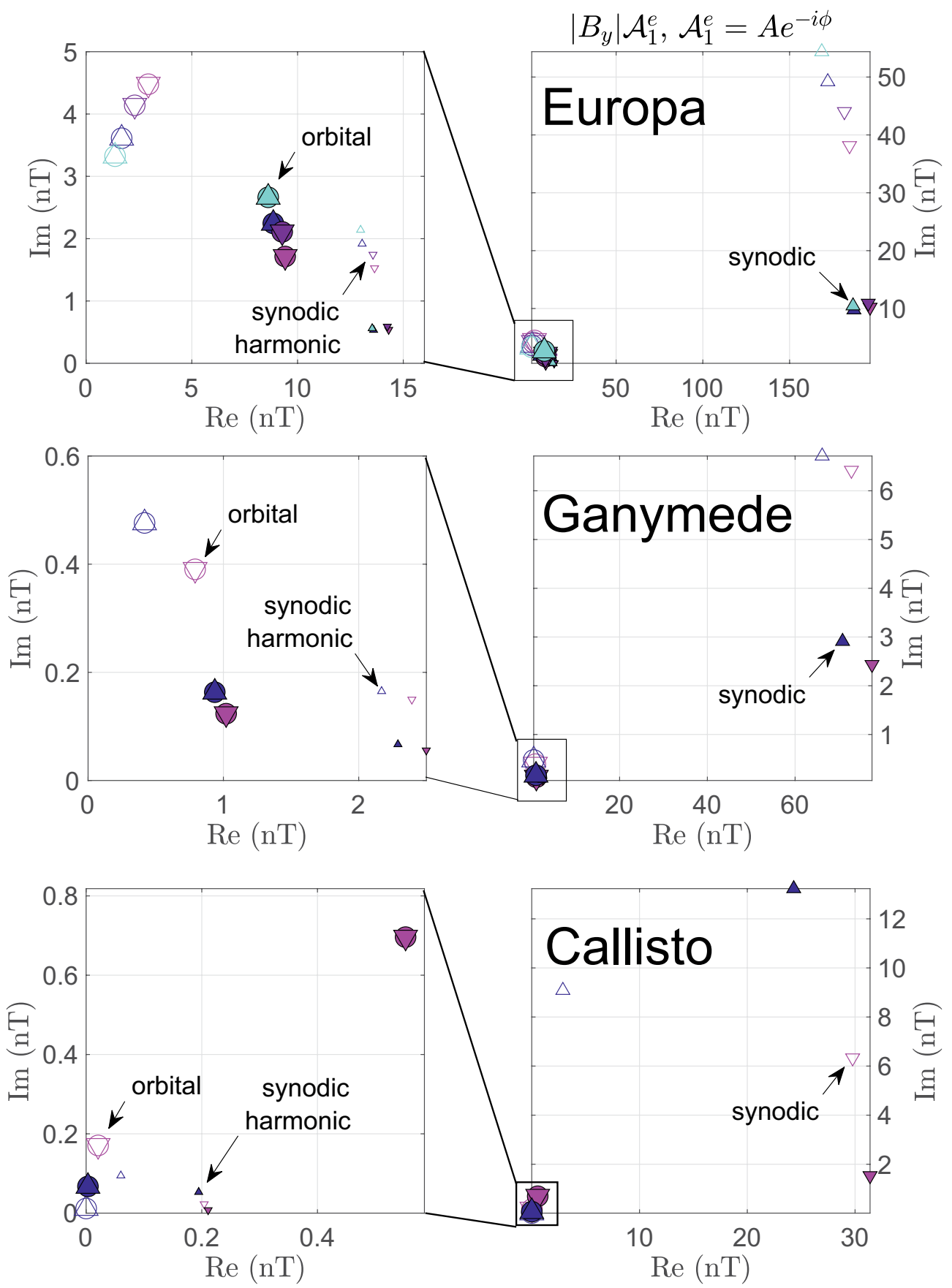

Figure 7. Real and imaginary components of the diffusive induction response to the changing $B_{y}$ component of Jupiter's magnetic field at the main driving periods (Figure 1) for \{Europa, Ganymede,Callisto\}. The real component (on the $x$-axis) is in phase with the excitation field, and the imaginary component (on the $y$-axis) is $90^{\circ}$ out-of-phase, as detailed in Section 2.6. Subpanels on the left side show the lower-magnitude signals of panels on the right. Filled symbols are for the higher concentrations. Upward and downward triangles are for thicker ice $(\{30,95,130\} \mathrm{km})$ and thinner ice $(\{5,26,100\} \mathrm{km})$, respectively. Symbol sizes scale with the period of the oscillation, denoting the orbital (largest), the synodic (intermediate), and the synodic harmonic (smallest). Circles are added to the orbital periods to guide the eye. 
the in-phase synodic component of the more saline and thick ocean with the less-saline, thin ocean. The imaginary component of the induced field $\left(B_{y} A \sin \phi\right)$ reveals the influence of the stronger phase delay for the lower-salinity oceans (Figure 7, empty symbols). The out-of-phase synodic signal in particular separates the $\mathrm{MgSO}_{4}$ and seawater models of constant ice thickness by $6 \mathrm{nT}$ for the lower-salinity models. For the 5 and $30 \mathrm{~km}$ ice thickness models, for fixed ocean composition, the separation of the stronger in-phase synodic components is 9 and $13 \mathrm{nT}$ for the nominal and lower-salinity models. The synodic harmonic components differ with salinity by as much as $1.5 \mathrm{nT}$ in the out-of-phase response, and by at most $0.7 \mathrm{nT}$ with ice thickness in the in-phase component.

The modeled Pedersen ionosphere has a maximum induction response of about $0.7 \mathrm{nT}$ in the out-of-phase synodic component Table 2. This is significant relative to the numerical precision of the calculation of about $0.001 \%$ (Figure S2). Including the ionosphere with the modeled adiabatic ocean conductivity profiles changes $B_{y}\{\operatorname{Re}, \operatorname{Im}\}\left(\mathcal{A}_{n}^{e}\right)$ less than $0.05 \mathrm{nT}$. Distinguishing such signal differences in spacecraft measurements of the magnetic field requires a very careful accounting of the fields generated by plasma, which is beyond the scope of this work.

Comparing the ocean with uniform conductivity set to the mean of the adiabatic profile $\bar{\sigma}$ with the adiabatic conductivity profile, the differences in the amplitude of the response field at the surface are as much as $0.7 \mathrm{nT}(0.4 \%)$ and $0.3 \mathrm{nT}(0.7 \%)$ for the synodic and orbital periods. For the uniform ocean using the conductivity at the ice-ocean interface $\sigma_{\text {top }}$, the orbital-period signal $(85.20 \mathrm{~h})$ differs by up to $20 \%$ for the warmer and lower-salinity oceans, or about $0.5 \mathrm{nT}$.

\subsubsection{Ganymede}

The synodic component separates the modeled ice thicknesses of 25 and $90 \mathrm{~km}\left(D_{\text {ocean }} \sim 450\right.$ and $\left.280 \mathrm{~km}\right)$ by about $7 \mathrm{nT}$ in the in-phase $B_{y}$ component, and for the nominal- and low-salinity models (10 and $1 \mathrm{wt} \%$ $\mathrm{MgSO}_{4}$ ) by about $4 \mathrm{nT}$ in both the in- the out-of-phase components. The orbital and synodic harmonic components show a similar pattern, with separations of about 0.2 and $0.1 \mathrm{nT}$.

Ganymede's ionospheric conductivity is smaller than Europa's. The resulting induction response is a maximum of about $0.03 \mathrm{nT}$, which adds small contributions to the oceanic fields that are comparable to the numerical resolution of the calculation. Currents and fields generated from Ganymede's magnetospheric interaction with Jupiter's local magnetic field and plasma are not considered in this calculation, although they may affect the induction signal (Paty \& Winglee, 2006; Jia et al., 2009; Payan et al., 2015). Hence, this work represents an end-member induction calculation, with contributions from Ganymede's magnetospheric current system left for future studies.

The uniformly conducting ocean with conductivity set to the mean of the adiabatic profile $\bar{\sigma}$ differs from the adiabatic profile in the amplitude of the response field at the surface by up to $1.2 \mathrm{nT}(1 \%)$ and $0.03 \mathrm{nT}(2 \%)$ at the synodic and orbital periods (Table 3 and Figure S4). The uniform ocean using the conductivity at the outermost ice-ocean interface $\sigma_{\text {top }}$ differs from the adiabatic case by up to $0.18 \mathrm{nT}(2 \%)$ for the orbital period.

\subsubsection{Callisto}

The synodic component shows different offsets for the thick/thin ice/ocean $(130 / 20 \mathrm{~km})$ and thinner ice/ thicker ocean $(100 / 130 \mathrm{~km})$ for the two examined $\mathrm{MgSO}_{4}$ compositions $(\{1,10\} \mathrm{wt} \%)$. For the thinner ice (downward arrows), the in-phase synodic components differ by $1.6 \mathrm{nT}$, while the out-of-phase components differ by nearly $5 \mathrm{nT}$. Models with thicker ice (upward arrows) have larger phase delays as well as larger separations in their amplitudes at the synodic period, creating a stronger in-phase separation of $21.4 \mathrm{nT}$, and a weaker out-of-phase separation of $4.1 \mathrm{nT}$. The synodic component has a similar configuration for the amplitude and phase responses, being close in period to the synodic period, and thus shows a similar pattern of separations as the synodic signal, albeit with smaller magnitudes on the order of $0.1 \mathrm{nT}$. The orbital component has stronger separation in both amplitude and phase for the thinner ice models, leading to proportionally larger differences in the induced field strengths, albeit for small overall magnitudes approaching zero except for the thin ice/thick ocean model that has a high-salinity.

Both the Pedersen and Cowling ionospheres have strong induced field strengths and affect the induction in the presence of and ocean. For the thick-ice/thin-ocean case with low salinity, the presence of the modeled 
ionospheres creates signals of comparable or much greater magnitude than the signal of the ocean by itself. In the Cowling case, the phase responses become reversed, such that the stronger field occurs for the inphase component. Comparing these different models, the influence of the oceans creates distinct in- and out-of-phase induction responses, such that with sufficient knowledge of the properties of the ionosphere it might be possible to infer the presence of an ocean.

The uniformly conducting ocean with conductivity set to the mean of the adiabatic profile $\bar{\sigma}$ differs from the adiabatic profile in the amplitude of the response field at the surface at the orbital period ( $400.33 \mathrm{~h})$ by $\lesssim 2$ pT. The induction responses of the $\sigma_{\text {top }}$ ocean models differ by up to 8 pT (10\%-20\%) for the orbital period.

\section{Motional Induction due to Ocean Convection}

We next consider motional induction driven by fluid flows within the oceans, which further complicates the interpretation of magnetic measurements. This effect is treated independently of the diffusive response considered above as a first approximation. Future work should consider the coupled induction response. Previous work by Tyler (2011) considered the possibility of magnetic remote sensing to detect resonant ocean tides on Europa in the limits of shallow water equations and thin-shell electrodynamics. Here, we focus instead on global fluid motions that may be driven by thermal convection within the oceans of Europa, Ganymede, and Callisto in the low-magnetic-Reynolds-number approximation in order to estimate upper bounds for motionally induced magnetic field amplitudes.

Thermal convection in icy satellite oceans is expected in order to efficiently transport heat from the deeper interior that arises primarily from radiogenic and tidal heating in the mantle (e.g., Soderlund et al., 2020). Using global convection models in combination with rotating convection theory, Soderlund et al. (2014) and Soderlund (2019) predicted the ocean of Europa to have large-scale flows organized into three zonal jets with retrograde (westward) flow at low latitudes and prograde (eastward) flow at high latitudes (Figure 8a). Upwelling at the equator and downwelling at mid to high latitudes effectively forms an overturning Hadley-like cell in each hemisphere (Figures 8b and 8c). Nonaxisymmetric convective motions are quasi-three-dimensional, due to rotational and inertial timescales of the flow being comparable. Predictions for Ganymede are significantly more uncertain, but Soderlund (2019) argued that a similar configuration may be expected. Convection in a possible Callisto ocean may be in the double-diffusive regime (Vance \& Brown, 2005; Vance \& Goodman, 2009) if the ocean's salt concentration is nearly saturated (Vance et al., 2018). However, considering thermal convection as an upper bound, application of the scaling arguments in Soderlund (2019) to Callisto suggest similar ocean flows here as well. The nominal ocean model shown in Figure 8 will, therefore, be assumed for all three ocean worlds considered here, noting that the use of nondimensional units permits different physical properties to be assumed for each satellite.

Because the modeled velocity field is given in units of the dimensionless Rossby number $R o=U / \Omega D$ (the ratio of rotational to inertial timescales), the results can be scaled to the different satellites with assumptions about ocean thickness $D$ and rotation rate $\Omega$ (Figure 8). A range of different ocean compositions, and therefore ocean thicknesses, are considered for velocity estimates that are given in Table 5. Flows are fastest for Ganymede and Europa, where the zonal jets can reach m/s speeds, the mean latitudinal flows have peak speeds of tens of $\mathrm{cm} / \mathrm{s}$, and the mean radial flows are $\sim 10 \mathrm{~cm} / \mathrm{s}$. At Callisto, flow speeds tend to be roughly an order of magnitude weaker.

Characteristic flow speeds $U$, in combination with the physical ocean properties $\sigma$ and $D$, allow the ratio of magnetic induction to magnetic diffusion to be estimated via the magnetic Reynolds number: $R m=\mu_{0} \sigma U D$. Using the values of these parameters from Table $5, R m \lesssim 1$ such that the low-magnetic-Reynolds approximation may be applied (Davidson, 2016). Here, the magnetic field $\mathbf{b}$ associated with induced current $\mathbf{J} \sim \sigma \mathbf{u} \times \mathbf{B}$ (Ohm's Law) due to velocity field $\mathbf{u}$ is small compared to the imposed magnetic field $\mathbf{B}_{o}$. Using Ampere's Law, the mean motionally induced field strength in the ocean can be estimated as

$$
b \sim \mu_{0} \sigma D U B_{o} \sim R m B_{o} .
$$

The resulting induced magnetic fields are thus stronger for larger electrical conductivities, ocean thicknesses, flow velocities, and satellites closer to the host planet since $B_{o}$ decreases with distance as $B_{o}=\{420$, 
(a) Model Geometry

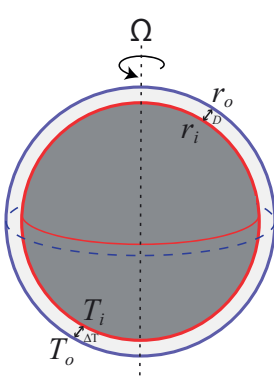

Dimensionless, $R o=U / \Omega D$

Dimensional, Europa $\left(\Omega=2.1 \cdot 10^{-5} \mathrm{~s}^{-1}, D=110 \mathrm{~km}\right)$

Dimensional, Ganymede $\left(\Omega=1.0 \cdot 10^{-5} \mathrm{~s}^{-1}, D=370 \mathrm{~km}\right)$

Dimensional, Callisto $\left(\Omega=4.4 \cdot 10^{-6} \mathrm{~s}^{-1}, D=75 \mathrm{~km}\right)$ (b) Zonal Flow, $u_{0}$

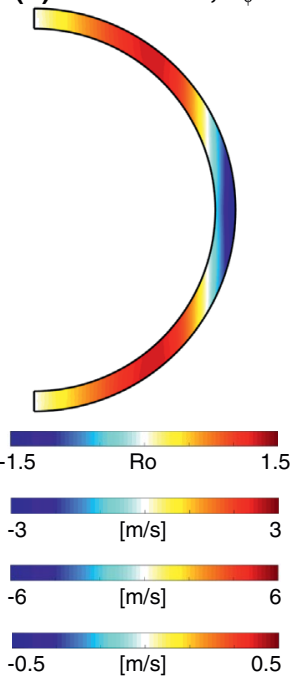

(c) Meridional Flow, $u_{\theta}$

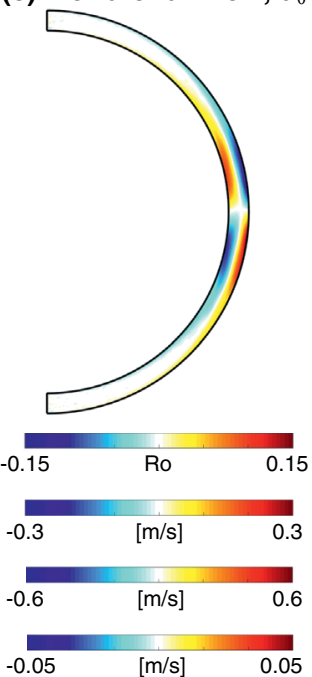

(d) Radial Flow, $u_{r}$

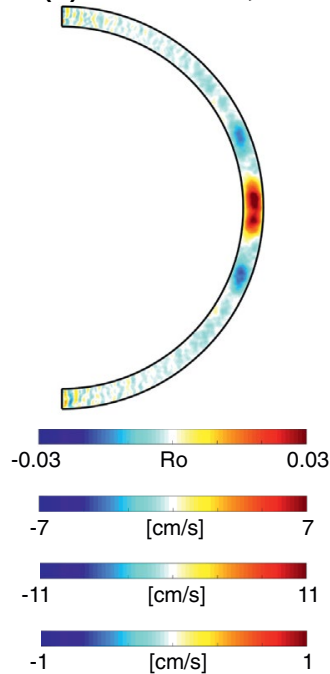

Figure 8. Mean flow fields in our nominal global ocean model from Soderlund (2019), averaged over 18 planetary rotations and all longitudes. To the previous work we add dimensional units for Europa, Ganymede, and Callisto assuming intermediate ocean thicknesses across the range of interior models, and a depiction of the meridional flow. (a) Geometry of the 3D ocean model. (b) Zonal (east-west) velocity field where red denotes prograde flows and blue denotes retrograde flows. (c) Meridional (latitudinal) velocity field where red denotes away from the north pole and blue denotes toward the north pole. (d) Radial velocity field where red denotes upwelling flows and blue denotes downwelling flows. The model has the following dimensionless input parameters: shell geometry $\chi=r_{i} / r_{o}=0.9$, Prandtl number $\operatorname{Pr}=\nu / \kappa=1$, Ekman number $E=\nu / \Omega D^{2}=3.0 \times 10^{-4}$, and Rayleigh number $R a=\alpha g \Delta T D^{3} / \nu \kappa$, where $r_{i}$ and $r_{o}$ are the inner and outer radii of the ocean, $D=r_{o}-r_{i}$ is ocean thickness, $\Omega$ is rotation rate, $\nu$ is kinematic viscosity, $\kappa$ is thermal diffusivity, $\alpha$ is thermal expansivity, $g$ is gravitational acceleration, and $\Delta T=T_{i}-T_{0}$ is the superadiabatic temperature contrast. The boundaries are impenetrable, stress-free, and isothermal.

120, 35\} nT for \{Europa, Ganymede,Callisto\} (Showman \& Malhotra, 1999). Ganymede is a special case because of its intrinsic magnetic field with surface field strength of $720 \mathrm{nT}$ at the equator and approximately twice that near the poles (Kivelson et al., 2002); thus, we assume here $B_{o} \approx 1,000 \mathrm{nT}$ as a mean value. Note that a more rigorous derivation of this relationship is given in Section S2, which demonstrates that these $b$ estimates should be taken as loose upper bounds and further distinguishes contributions due to radial, meridional, and azimuthal velocity fields.

Table 5 summarizes the assumed ocean flows at Europa, Ganymede, and Callisto as well as estimates of their induced magnetic field strengths at the top of the ocean. Field strengths at the surface will be a factor of $\left(r_{\text {ocean }} / r_{\text {satellite }}\right)^{(l+2)}$ times weaker, where $l$ is spherical harmonic degree, so the surface fields will be weaker by $\lesssim\{6 \%, 10 \%, 15 \%\}$ at \{Europa, Ganymede,Callisto $\}$ assuming a dipole $l=1$ configuration for the most optimistic amplitude. Our analysis focuses on the radial $b_{r} \sim R m_{r} B_{o}$ component because boundary-confined surface currents can cause discontinuities in the tangential induced magnetic components. We also assume flow speeds typical of the steady overturning cells due to their temporal persistence and large spatial scale, which we hypothesize will produce the strongest induced magnetic signatures and would be more easily discernible by spacecraft. We find that $b_{r} \lesssim 20 \mathrm{nT}$ for Europa, $b_{r} \lesssim 300 \mathrm{nT}$ for Ganymede, and $b_{r} \lesssim 1 \mathrm{nT}$ for Callisto. Implications of these field estimates on magnetic measurements and future work needed for their refinement are discussed in the next section.

\section{Discussion and Conclusions}

The inverse problem of reconstructing the full induction response from spacecraft data is beyond the scope of this work, and is discussed in detail elsewhere (e.g., Khurana et al., 2009, and Cochrane et al. in progress). We focus here on the significance and separability of the diffusive induction responses for the physically consistent models described above. We examine the likelihood of being able to detect and separate the signals of motional induction from the diffusive signals. We also discuss the merits of using physically con- 
Table 5

Ocean Characteristics and Upper Bound Estimates of the Motionally Induced Magnetic Field Strengths From Equation (12) at the top of the oceans

\begin{tabular}{|c|c|c|c|c|c|c|}
\hline & $\sigma[\mathrm{S} / \mathrm{m}]$ & $D[\mathrm{~km}]$ & $U_{r}[\mathrm{~m} / \mathrm{s}]$ & $U_{\theta}[\mathrm{m} / \mathrm{s}]$ & $U_{\phi}[\mathrm{m} / \mathrm{s}]$ & $b_{r}[\mathrm{nT}]$ \\
\hline \multicolumn{7}{|l|}{ Europa } \\
\hline $\mathrm{MgSO}_{4} 1 \mathrm{wt} \%$, Thicker ice shell & 0.4 & 91 & 0.06 & 0.29 & 2.9 & 1 \\
\hline $\mathrm{MgSO}_{4} 1 \mathrm{wt} \%$, Thinner ice shell & 0.5 & 117 & 0.07 & 0.37 & 3.7 & 2 \\
\hline $\mathrm{MgSO}_{4} 10 \mathrm{wt} \%$, Thicker ice shell & 3.4 & 96 & 0.06 & 0.30 & 3.0 & 10 \\
\hline $\mathrm{MgSO}_{4} 10 \mathrm{wt} \%$, Thinner ice shell & 3.8 & 124 & 0.08 & 0.39 & 3.9 & 20 \\
\hline Seawater 0.35 wt $\%$, Thicker ice shell & 0.4 & 91 & 0.06 & 0.29 & 2.9 & 1 \\
\hline Seawater $0.35 \mathrm{wt} \%$, Thinner ice shell & 0.4 & 117 & 0.07 & 0.37 & 3.7 & 2 \\
\hline Seawater $3.5 \mathrm{wt} \%$, Thicker ice shell & 2.9 & 91 & 0.06 & 0.29 & 2.9 & 8 \\
\hline Seawater $3.5 \mathrm{wt} \%$, Thinner ice shell & 3.1 & 119 & 0.07 & 0.37 & 3.7 & 14 \\
\hline \multicolumn{7}{|l|}{ Ganymede } \\
\hline $\mathrm{MgSO}_{4} 1 \mathrm{wt} \%$, Thicker ice shell & 0.3 & 276 & 0.08 & 0.41 & 4.1 & 8 \\
\hline $\mathrm{MgSO}_{4} 1 \mathrm{wt} \%$, Thinner ice shell & 0.5 & 442 & 0.13 & 0.66 & 6.6 & 36 \\
\hline $\mathrm{MgSO}_{4} 10 \mathrm{wt} \%$, Thicker ice shell & 2.3 & 282 & 0.08 & 0.42 & 4.2 & 65 \\
\hline $\mathrm{MgSO}_{4} 10 \mathrm{wt} \%$, Thinner ice shell & 4.1 & 458 & 0.14 & 0.69 & 6.9 & 330 \\
\hline \multicolumn{7}{|l|}{ Callisto } \\
\hline $\mathrm{MgSO}_{4} 1 \mathrm{wt} \%$, Thicker ice shell & 0.09 & 21 & 0.003 & 0.01 & 0.14 & $\ll 1$ \\
\hline $\mathrm{MgSO}_{4} 1 \mathrm{wt} \%$, Thinner ice shell & 0.2 & 132 & 0.02 & 0.09 & 0.87 & 0.02 \\
\hline $\mathrm{MgSO}_{4} 10 \mathrm{wt} \%$, Thicker ice shell & 0.6 & 21 & 0.002 & 0.01 & 0.12 & $\ll 1$ \\
\hline $\mathrm{MgSO}_{4} 10 \mathrm{wt} \%$, Thinner ice shell & 1.5 & 130 & 0.02 & 0.09 & 0.86 & 0.2 \\
\hline
\end{tabular}

Note. Radial $\mathrm{U}_{\mathrm{r}}$, latitudinal $\mathrm{U}_{\theta}$, and zonal $\mathrm{U}_{\phi}$ flow speeds from Figure 8 with $\mathrm{U}=\Omega \mathrm{DR}_{0}$; ocean thicknesses $\mathrm{D}$ and electrical conductivity $\sigma$ from Tables $2-4$.

sistent models as inputs to the inverse problem, the future experimental and modeling work that is needed for material properties and motional induction, and the implications for future missions.

\subsection{Significance and Separability of the Diffusive and Motional Signals}

The representative, physically consistent structures of Jupiter's ocean moons that we model have distinct magnetic induction signals when the phase delays are considered. The waveform responses at the three characteristic periods identified for each moon (Figure 7; Tables 2-4) illustrate the possibility for inferring key properties of the moons, possibly by planning missions (Section 4.3). This study demonstrates the existence of magnetic induction responses tracing to the unique melting curves of different ocean compositions, and thus to physical features arising from their coupled thermal and chemical evolution. Lower salinity oceans have larger induced responses that are out-of-phase with Jupiter's rotating field.

For Europa, models consistent with reducing/oxidizing $\left(\mathrm{MgSO}_{4}-/ \mathrm{NaCl}\right.$-dominated) oceans have distinct induction features at all three periods considered here. We find a motionally induced field of $b_{r} \lesssim 20 \mathrm{nT}$ for Europa, or up to $5 \%$ of the ambient jovian field. For comparison, the field strength induced by tidal motions (Rossby-Haurwitz response to obliquity tidal forcing) is $\sim 1 \mathrm{nT}$ (Tyler, 2011) and at Jupiter's synodic period of $11.23 \mathrm{~h}$ is $\lesssim 200 \mathrm{nT}$ (Figure 7; Table 2). Schilling et al. (2004) found an upper limit for an intrinsic magnetic field at Europa to be $25 \mathrm{nT}$ at the surface, implying that an observable signal from motional flows may have gone unnoticed there. A detailed analysis is required to better characterize the potential response and its implications for determining ocean composition, salinity, and convective flows.

For Ganymede, the tabulated results (Table 3) show that a plausible liquid layer at the rock interface beneath the high-pressure ice would create an in-phase signal of about $0.01 \mathrm{nT}$ at the orbital period. The ionosphere should not impede sensing the induction response of the ocean. Here, $b_{r} \lesssim 300 \mathrm{nT}$, which approaches half of the equatorial surface strength of the satellite's intrinsic field for the thickest, saltiest ocean 
considered; magnetic fields induced at Jupiter's synodic period of $10.53 \mathrm{~h}$ are $\lesssim 80 \mathrm{nT}$ (Figure 7; Table 3). As a result, these motionally induced magnetic fields warrant further study as they may allow ocean flows to be inferred, bias electrical conductivity inversions, and/or complicate extraction of Ganymede's core dynamo magnetic field component.

For Callisto, strong induction responses ( $>10 \mathrm{nT})$ that might be used to infer the ocean's conductivity and thickness might exist at the synodic period of Jupiter's rotation, with smaller signals $(>1 \mathrm{nT})$ at the other excitation periods. However, the modeled Cowling ionosphere without any ocean creates a strong induction response that is not easily distinguished from an oceanic signal. Motional inductions signals of $b_{r} \lesssim 1 \mathrm{nT}$ are less significant relative to the peak strength $(\lesssim 30 \mathrm{nT})$ of the field induced at Jupiter's synodic period of $10.18 \mathrm{~h}$ (Figure 7; Table 4). Thus, as demonstrated and further discussed by Hartkorn and Saur (2017), magnetic induction measured by the Galileo spacecraft (Kivelson et al., 1999) might be explained as resulting from the response of Callisto's ionosphere and not an ocean.

Structural models of ocean worlds (e.g., Schubert et al., 2004) often assume a uniform ocean temperature determined by the melting temperature of the ice-ocean interface. Using this temperature as the basis for the ocean's electrical conductivity leads to large differences from the more physically consistent, adiabatic case. The greater mismatch of conductivities of the lower part of the ocean causes large differences in amplitude and phase at longer periods (i.e. for larger skin depths).

Prior analyses of magnetic induction in Jupiter's ocean moons have all assumed a uniform conductivity of the oceans (Khurana et al., 2002; Kivelson et al., 2002, 2000; Schilling et al., 2007; Seufert et al., 2011). For all three moons, we compared the diffusive response for a uniformly conducting ocean with conductivity set to a reference value from the adiabatic conductivity profile. We find that the diffusive induction responses of the oceans with uniform conductivity equal to the mean of the adiabatic profile are, for many interior configurations, a reasonable approximation to the induction response for a more realistic electrical conductivity following the adiabatic profile. The response amplitudes are most distinct between the adiabatic and mean-conductivity oceans for the thin-ice, lower-salinity configurations.

For the mean-conductivity oceans $(\bar{\sigma})$, the in-phase response amplitudes are all larger than for the corresponding adiabatic profiles and the out-of-phase amplitudes mostly decrease slightly (see Tables 2-4).

For Europa, the in-phase response amplitudes range from about $0.22 \%$ to $0.46 \%$ greater for the synodic period and from $0.28 \%$ to $1.02 \%$ greater for the orbital period; the out-of-phase responses range from $2.87 \%$ less to $0.03 \%$ greater for the synodic period and from $0.10 \%$ less to $0.63 \%$ greater for the orbital period. Larger differences are observed for thinner-ice, warmer oceans in all cases.

For Ganymede, the in-phase response amplitudes range from about $0.38 \%$ to $1.23 \%$ greater for the synodic period and from $1.01 \%$ to $2.61 \%$ greater for the orbital period; the out-of-phase responses range from $9.78 \%$ to $2.65 \%$ less for the synodic period and from $3.07 \%$ less to $1.41 \%$ greater for the orbital period. These excesses/deficits in the synodic/orbital component differences arise because the mean conductivity case increases/reduces the conductance contributed by the shallower/deeper parts of the ocean (Figure 5) associated with smaller/larger skin depths of the diffusive response.

For Callisto, the in-phase response amplitudes range from $0.00 \%$ to $0.53 \%$ greater for the synodic period and from $0.00 \%$ to $1.45 \%$ greater for the orbital period; the out-of-phase responses range from $1.74 \%$ less to $0.03 \%$ greater for the synodic period and from $0.00 \%$ to $0.96 \%$ greater for the orbital period. For the thicker oceans, where conductivity changes with depth, the differences are similar to those for Ganymede.

We also considered the diffusive response from uniformly conducting oceans with a conductivity equal to that at the ice-ocean interface $\left(\sigma_{\text {top }}\right)$ in comparison to the adiabatic profiles (see Tables $\left.2-4\right)$. Unlike the mean-conductivity oceans, there is not a consistent pattern of larger or smaller responses when compared to the adiabatic case.

For Europa, the in-phase response amplitudes range from about $1.49 \%$ less to $0.10 \%$ greater for the synodic period and from $16.33 \%$ to $0.34 \%$ less for the orbital period; the out-of-phase responses range from $2.13 \%$ to $10.77 \%$ greater for the synodic period and from $5.92 \%$ less to $11.33 \%$ greater for the orbital period. Differences are consistently large in this comparison. 
For Ganymede, the in-phase response amplitudes range from about $0.14 \%$ less to $0.45 \%$ greater for the synodic period and from $22.82 \%$ to $0.11 \%$ less for the orbital period; the out-of-phase responses range from $2.51 \%$ less to $10.74 \%$ greater for the synodic period and from $3.32 \%$ less to $17.09 \%$ greater for the orbital period. For the lower-salinity ocean we model, the marked difference in phase delay between the thin-ice, warmer profile and the thick-ice, colder profile (Figure 6) is evident in how the in-phase and out-of-phase components change between the two cases.

For Callisto, the in-phase response amplitudes range from about $4.12 \%$ less to $0.28 \%$ greater for the synodic period and from $26.08 \%$ less to $1.23 \%$ greater for the orbital period; the out-of-phase responses range from $1.87 \%$ less to $15.03 \%$ greater for the synodic period and from $13.62 \%$ less to $0.61 \%$ greater for the orbital period. The lower phase lag of the nominal salinity case for the thicker ocean is evident in the differences between the in-phase and out-of-phase components from the other cases.

For larger oceans, where the nonlinear pressure behavior of the adiabat introduces curvature to the electrical conductivity profile, slightly larger differences can arise for thicker oceans. The presence of high-pressure ice also enhances the sensitivity of the overall ocean thickness to the ocean's salinity.

\subsection{Future Experimental and Modeling Work}

The diffusive induction models described in Section 2.3 make use of thermodynamic and electrical conductivity data developed for applications to ocean worlds (Vance \& Brown, 2013; Vance et al., 2018). Future work should explore a broader space of compositions. Constructing models that account for the effects of high concentration and pressure requires updated thermodynamic data (Bollengier et al., 2019; Journaux et al., 2020), as described above, matched with accurate electrical conductivity data. Recent progress in applying electrical conductivity to geochemical systems at Earth's surface (McCleskey et al., 2012) provides a starting point for considering oceanic concentrations with realistic assemblages of salts (Kargel et al., 2000; Zolotov \& Shock, 2001). Extending these data to high-pressures and concentrations requires further experimental work (e.g., Guo \& Keppler, 2019; Keppler, 2014). Future investigations should also examine a fuller parameter space of interior structures, including conductivity in the solid layers. Such future work should examine a broader range of ice and hydrosphere thicknesses, including density structures that explore the full range of constraints based on Galileo gravity data, not just the mean values of the moments of inertia (Schubert et al. 2004; Vance et al., 2019). Future work should also examine asymmetry in the conducting layers. Work by Seufert et al. (2011) permits consideration of small deviations from spherical symmetry, for example due to long-wavelength variations in the thickness of Europa's ice (Nimmo et al., 2007). Ultimately, the ability to consider diffusive magnetic induction from electrically conducting regions with arbitrary geometry would enable accounting for the effects of the Cowling ionosphere at Callisto (Hartkorn \& Saur, 2017), meridional variations in salinity at Europa (Zhu et al., 2017), brine lenses in Europa's ice (Schmidt et al., 2011).

The simplified approaches to motional induction described in Sections 3 and S2 give order of magnitude estimates of the maximum induced fields due to ocean convection and shows that these fields may be large enough to impact interpretations of magnetic measurements. Future work will assess the implications of the simplifying assumptions made through more detailed calculations. For example, we have assumed homogeneous and constant jovian and Ganymede background fields. However, the temporal and spatial variation of the ambient fields and the plasma interaction fields generated by currents (especially Ganymede's magnetosphere) are expected to be significant, and the magnetic environment each satellite experiences throughout its orbit is highly dynamic (e.g., Bagenal et al., 2015). The influence of these variations on oceanflow-driven magnetic field signatures also remains to be explored (cf. Gissinger \& Petitdemange, 2019). Kinematic models that directly solve the coupled momentum and induction equations to determine the motionally induced magnetic fields are an exciting and necessary future venue to refine these estimates. The resulting predictions for field strength and spatial structure may allow the motional and diffusive components of the induced magnetic field to be separated, facilitating better electrical conductivity inversions and ocean flow hypothesis tests. 


\subsection{Implications for Future Missions}

The Europa Clipper mission will conduct multiple ( $>40)$ flybys of Europa, and will investigate its magnetic induction response with the goal of constraining the ocean salinity and ice thickness, each to within $50 \%$. With independent constraints on ice thickness obtained from the Radar for Europa Assessment and Sounding: Ocean to Near-surface (REASON) and Europa Imaging System (EIS) investigations (Steinbrügge et al., 2018), it may be possible to constrain the ocean's temperature and thus the adiabatic structure for the best-fit ocean composition inferred from compositional investigations. The analyses provided here (Figure 7 and Table 2) indicate that a sensitivity of $1.5 \mathrm{nT}$ is probably sufficient to distinguish between the end-member $\mathrm{MgSO}_{4}$ and $\mathrm{NaCl}$ oceans, and the corresponding ice thicknesses considered here.

The JUpiter ICy moons Explorer (JUICE) mission will execute two Europa flybys and nine Callisto flybys, and will orbit Ganymede (Grasset et al., 2013). The magnetic field investigation seeks to determine the induction response to better than $0.1 \mathrm{nT}$. The Europa flybys might aid the Europa Clipper investigation in constraining the composition of the ocean. We find that at Ganymede, JUICE's magnetic field investigation will not be sufficient to discern the modeled basal liquid layer at the ice VI-rock interface, which would require sensitivity better than $0.01 \mathrm{nT}$. Although the ability to discern between ocean compositions could not be assessed owing to insufficient thermodynamic and electrical conductivity data at high pressures, it seems likely that useful constraints could be derived based on the signal strengths at Ganymede, if appropriate laboratory-derived data for relevant solutions under pressure became available. Motional induction also appears to be even more important to consider at Ganymede than Europa, with JUICE's orbital tour providing a continuous and detailed data set at Ganymede, critical in investigating the interplay and relative contributions of magnetospheric interaction fields, diffusive induction fields, and motional induction fields.

At Callisto, both Europa Clipper and JUICE would be able to investigate the synodic signals that vary by more than $2 \mathrm{nT}$ for the different models considered here, including models with only an ionosphere. JUICE's $0.1 \mathrm{nT}$ sensitivity might be able to obtain useful information at the orbital and first harmonic periods as well. In contrast with Europa and Ganymede, however, good knowledge of the ionospheric structure at Callisto is required for detecting an ocean.

\section{Data Availability Statement}

The Matlab scripts and associated data needed to compute the results of this work are archived as a release on github (https://github.com/vancesteven/PlanetProfile) with DOI: 10.5281/zenodo.4052711. All global ocean convection model data were first published in Soderlund (2019) and are available therein.

Acknowledgments

Work by JPL co-authors was partially supported by the Jet Propulsion Laboratory, Caltech, and by the Icy Worlds node of NASA's Astrobiology Institute (13-13NAI7_2-0024). This work was partially supported by NASA's Europa Clipper project. Work by M. J. Styczinski was supported by the NASA Earth and Space Science Fellowship Program-Grant 80NSSC18K1236. Work by K. M. Soderlund was also supported by NASA Grant NNX14AR28G.

\section{References}

Acuna, M. H., \& Ness, N. F. (1976). The main magnetic field of jupiter. Journal of Geophysical Research, 81(16), 2917-2922. https://doi. org/10.1029/ja081i016p02917

Bagenal, F., Sidrow, E., Wilson, R. J., Cassidy, T. A., Dols, V., Crary, F. J., et al. (2015). Plasma conditions at Europa's orbit. Icarus, 261 , 1-13. https://doi.org/10.1016/j.icarus.2015.07.036

Bollengier, O., Brown, J. M., \& Shaw, G. H. (2019). Thermodynamics of pure liquid water: Sound speed measurements to $700 \mathrm{MPa}$ down to the freezing point, and an equation of state to $2300 \mathrm{MPa}$ from 240 to 500K. The Journal of Chemical Physics, 151(5), 054501. https:// doi.org/10.1063/1.5097179

Buffington, B., Lam, T., Campagnola, S., Ludwinski, J., Ferguson, E., Bradley, B., et al. (2017). Evolution of trajectory design requirements on NASA's planned Europa Clipper mission. Proceedings of the 68th International Astronautical Congress (IAC)(IAC-17-C1.7.8). International Astronautical Congress.

Calvert, R., Cornelius, J. A., Griffiths, V. S., \& Stock, D. I. (1958). The determination of the electrical conductivities of some concentrated electrolyte solutions using a transformer bridge. Journal of Physical Chemistry, 62(1), 47-53.

Chave, A. D. (1983). On the theory of electromagnetic induction in the Earth by ocean currents. Journal of Geophysical Research, 88, 3531-3542.

Choblet, G., Tobie, G., Sotin, C., Kalousová, K., \& Grasset, O. (2017). Heat transport in the high-pressure ice mantle of large icy moons. Icarus, 285, 252-262. https://doi.org/10.1016/j.icarus.2016.12.002

Connerney, et al. (1998). New models of Jupiter's magnetic field constrained by the Io flux tube footprint. Journal of Geophysical Research, 103(A6), 11929-11939.

Connerney, J. E. P., Acuna, M., \& Ness, N. (1981). Modeling the Jovian current sheet and inner magnetosphere. Journal of Geophysical Research, 86(A10), 8370-8384.

Connerney, J. E. P., Kotsiaros, S., Oliversen, R. J., Espley, J. R., Joergensen, J. L., Joergensen, P. S., et al. (2018). A new model of Jupiter's magnetic field from Juno's first nine orbits. Geophysical Research Letters, 45(6), 2590-2596. https://doi.org/10.1002/2018gl077312

Constable, S., \& Constable, C. G. (2004). Observing geomagnetic induction in magnetic satellite measurements and associated implications for mantle conductivity. Geochemistry, Geophysics, Geosystems, 5, Q01006. https://doi.org/10.1029/2003GC000634 
Davidson, P. A. (2016). Introduction to magnetohydrodynamics (2nd ed.) Cambridge: Cambridge University Press. https://doi. org/10.1017/9781316672853

Eckhardt, D. H. (1963). Geomagnetic induction in a concentrically stratified earth. Journal of Geophysical Research, 68(23), 6273-6278. https://doi.org/10.1029/jz068i023p06273

Gissinger, C., \& Petitdemange, L. (2019). A magnetically driven equatorial jet in Europa's ocean. Nature Astronomy, 3, 401-407. https:// doi.org/10.1038/s41550-019-0713-3

Glein, C., Postberg, F., \& Vance, S. (2019). The geochemistry of Enceladus: Composition and controls (p. 39-56). Tucson: University of Arizona Press.

Grasset, O., Dougherty, M., Coustenis, A., Bunce, E., Erd, C., Titov, D., et al. (2013). JUpiter ICy moons Explorer (JUICE): An ESA mission to orbit Ganymede and to characterise the Jupiter system. Planetary and Space Science, 78, 1-21. https://doi.org/10.1016/j.pss.2012.12.002

Grayver, A. V., Schnepf, N. R., Kuvshinov, A. V., Sabaka, T. J., Manoj, C., \& Olsen, N. (2016). Satellite tidal magnetic signals constrain oceanic lithosphere-asthenosphere boundary. Science Advances, 2(9), e1600798. https://doi.org/10.1126/sciadv.1600798

Guo, H., \& Keppler, H. (2019). Electrical conductivity of NaCl-bearing aqueous fluids to $900^{\circ} \mathrm{C}$ and $5 \mathrm{GPa}$. Journal of Geophysical Research: Solid Earth, 124(2), 1397-1411. https://doi.org/10.1029/2018jb016658

Hand, K., \& Chyba, C. (2007). Empirical constraints on the salinity of the European ocean and implications for a thin ice shell. Icarus, 189(2), 424-438. 10.1016/j.icarus.2007.02.002

Hartkorn, O., \& Saur, J. (2017). Induction signals from Callisto's ionosphere and their implications on a possible subsurface ocean. Journal of Geophysical Research: Space Physics, 122(11), 11677-11697. https://doi.org/10.1002/2017ja024269

Hogenboom, D. L., Kargel, J. S., Ganasan, J. P., \& Lee, L. (1995). Magnesium sulfate-water to 400 MPa using a novel piezometer: Densities, phase equilibria, and planetological implications. Icarus, 115(2), 258-277. https://doi.org/10.1006/icar.1995.1096

Jia, X., Walker, R. J., Kivelson, M. G., Khurana, K. K., \& Linker, J. A. (2009). Properties of Ganymede's magnetosphere inferred from improved three-dimensional MHD simulations. Journal of Geophysical Research: Space Physics, 114(A9), A09209. https://doi. org/10.1029/2009JA014375

Journaux, B., Brown, J., Pakhomova, A., Collings, I., Petitgirard, S., Espinoza, P., et al. (2020). Holistic approach for studying planetary hydrospheres: Gibbs representation of ices thermodynamics, elasticity and the water phase diagram to $2300 \mathrm{MPa}$. Journal of Geophysical Research: Planets, 125, e2019JE006176. https://doi.org/10.1029/2019je006176

Journaux, B., Daniel, I., Caracas, R., Montagnac, G., \& Cardon, H. (2013). Influence of NaCl on ice VI and ice VII melting curves up to 6GPa, implications for large icy moons. Icarus, 226(1), 355-363. https://doi.org/10.1016/j.icarus.2013.05.039

Journaux, B., Daniel, I., Petitgirard, S., Cardon, H., Perrillat, J.-P., Caracas, R., \& Mezouar, M. (2017). Salt partitioning between water and high-pressure ices. implication for the dynamics and habitability of icy moons and water-rich planetary bodies. Earth and Planetary Science Letters, 463, 36-47. https://doi.org/10.1016/j.epsl.2017.01.017

Kalousová, K., Sotin, C., Choblet, G., Tobie, G., \& Grasset, O. (2018). Two-phase convection in Ganymede's high-pressure ice layer-implications for its geological evolution. Icarus, 299, 133-147. https://doi.org/10.1016/j.icarus.2017.07.018

Kargel, J. S., Kaye, J. Z., Head, J. W., III, Marion, G. M., Sassen, R., Crowley, J. K., (2000). Europa's crust and ocean: Origin, composition, and the prospects for life. Icarus, 148(1), 226-265. https://doi.org/10.1006/icar.2000.6471

Keppler, H. (2014). Electrical conductivity measurements of aqueous fluids under pressure with a hydrothermal diamond anvil cell. Review of Scientific Instruments, 85(11), 115107. https://doi.org/10.1063/1.4902152

Khurana, K. K. (1997). Euler potential models of Jupiter's magnetospheric field. Journal of Geophysical Research, 102(A6), 11295-11306. https://doi.org/10.1029/97ja00563

Khurana, K., et al. (2002). Searching for liquid water in Europa by using surface observatories. Astrobiology, 2(1), 93-103. https://doi. org/10.1089/153110702753621376

Khurana, K., Kivelson, M., Hand, K., \& Russell, C. (2009). Electromagnetic induction from Europa's ocean and the deep interior. Eds Robert T., Pappalardo, William B., McKinnon, Krishan K., Khurana; In Europa (Vol. 1, p. 571). with the assistance of René Dotson with 85 collaborating authors. Tucson, AZ: University of Arizona Press, The University of Arizona space science series ISBN: 9780816528448.

Khurana, K., Kivelson, M., Stevenson, D., Schubert, G., Russell, C., Walker, R., \& Polanskey, C. (1998). Induced magnetic fields as evidence for subsurface oceans in Europa and Callisto. Nature, 395(6704), 777-780.

Kivelson, M., Khurana, K., Russell, C., Volwerk, M., Walker, R., \& Zimmer, C. (2000). Galileo magnetometer measurements: A stronger case for a subsurface ocean at Europa. Science, 289, 1340-1343.

Kivelson, M., Khurana, K., Stevenson, D., Bennett, L., Joy, S., Russell, C., et al. (1999). Europa and Callisto: Induced or intrinsic fields in a periodically varying plasma environment. Journal of Geophysical Research, 104(A3), 4609-4625.

Kivelson, M., Khurana, K., \& Volwerk, M. (2002). The permanent and inductive magnetic moments of Ganymede. Icarus, 157(2), 507-522. https://doi.org/10.1006/icar.2002.6834

Lainey, V., Duriez, L., \& Vienne, A. (2006). Synthetic representation of the Galilean satellites' orbital motions from L1 ephemerides. Astronomy \& Astrophysics, 456, 783-788. https://doi.org/10.1051/0004-6361:20064941

Lieske, J. (1998). Galilean satellite ephemerides E5. Astronomy \& Astrophysics Supplement Series, 129(2), $205-217$.

Liuzzo, L., Feyerabend, M., Simon, S., \& Motschmann, U. (2015). The impact of Callisto's atmosphere on its plasma interaction with the jovian magnetosphere. Journal of Geophysical Research: Space Physics, 120(11), 9401-9427. https://doi.org/10.1002/2015ja021792

McCleskey, R. B., Nordstrom, D. K., Ryan, J. N., \& Ball, J. W. (2012). A new method of calculating electrical conductivity with applications to natural waters. Geochimica et Cosmochimica Acta, 77, 369-382. https://doi.org/10.1016/j.gca.2011.10.031

McDougall, T. J., \& Barker, P. M. (2011). Getting started with TEOS-10 and the Gibbs Seawater (GSW) oceanographic toolbox. SCOR/ IAPSO WG, 127, 1-28.

Melwani Daswani, M., Vance, S. D., Mayne, M. J., \& Glein, C. R.. A metamorphic origin for Europa's ocean. (under revision). Geophysical Research Letters.

Minami, T. (2017). Motional induction by tsunamis and ocean tides: 10 years of progress. Surveys in Geophysics, 38, 1097-1132. https://doi. org/10.1007/s10712-017-9417-3

Nimmo, F., Thomas, P., Pappalardo, R., \& Moore, W. (2007). The global shape of Europa: Constraints on lateral shell thickness variations. Icarus, 191(1), 183-192. https://doi.org/10.1016/j.icarus.2007.04.021

Parkinson, W. (1983). Introduction to geomagnetism. Edinburgh: Scottish Academic Press.

Pasek, M. A., \& Greenberg, R. (2012). Acidification of Europa's subsurface ocean as a consequence of oxidant delivery. Astrobiology, 12(2), 151-159. https://doi.org/10.1089/ast.2011.0666

Paty, C., \& Winglee, R. (2006). The role of ion cyclotron motion at Ganymede: Magnetic field morphology and magnetospheric dynamics. Geophysical Research Letters, 33(10), L10106. https://doi.org/10.1029/2005GL025273 
Payan, A., Paty, C., \& Retherford, K. (2015). Uncovering local magnetospheric processes governing the morphology and variability of Ganymede's aurora using three-dimensional multifluid simulations of Ganymede's magnetosphere. Journal of Geophysical Research: Space Physics, 120(1), 401-413. https://doi.org/10.1002/2014JA020301

Saur, J., Neubauer, F. M., \& Glassmeier, K.-H. (2009). Induced magnetic fields in solar system bodies. Space Science Reviews, 152(1-4), 391-421. https://doi.org/10.1007/s11214-009-9581-y

Saur, J., Strobel, D. F., \& Neubauer, F. M. (1998). Interaction of the jovian magnetosphere with europa: Constraints on the neutral atmosphere. Journal of Geophysical Research, 103(E9), 19947-19962. https://doi.org/10.1029/97je03556

Schilling, N. (2006). Time varying interaction of Europa's atmosphere-ionosphere and its conducting ocean with the Jovian magnetosphere, Cologne: (Unpublished doctoral dissertation). Universität zu Köln.

Schilling, N., Khurana, K., \& Kivelson, M. (2004). Limits on an intrinsic dipole moment in Europa. Journal of Geophysical Research, 109, E05006. https://doi.org/10.1029/2003JE002166

Schilling, N., Neubauer, F. M., \& Saur, J. (2007). Time-varying interaction of Europa with the jovian magnetosphere: Constraints on the conductivity of Europa's subsurface ocean. Icarus, 192(1), 41-55. https://doi.org/10.1016/j.icarus.2007.06.024

Schmidt, B., Blankenship, D., Patterson, G., \& Schenk, P. (2011). Active formation of chaos terrain over shallow subsurface water on Europa. Nature, 479(7374), 502-505. https://doi.org/10.1038/nature10608

Schmidt, C., \& Manning, C. (2017). Pressure-induced ion pairing in $\mathrm{MgSO}_{4}$ solutions: Implications for the oceans of icy worlds. Geochemical Perspectives Letters, 3, 66-74. https://doi.org/10.7185/geochemlet.1707

Schubert, G., Anderson, J., Spohn, T., \& McKinnon, W. (2004). Interior composition, structure and dynamics of the Galilean satellites. In Jupiter: The Planet, Satellites and Magnetosphere, 281-306. Cambridge University Press.

Seufert, M., Saur, J., \& Neubauer, F. M. (2011). Multi-frequency electromagnetic sounding of the Galilean moons. Icarus, 214(2), 477-494. https://doi.org/10.1016/j.icarus.2011.03.017

Showman, A. P., \& Malhotra, R. (1999). The Galilean satellites. Science, 286(5437), 77-84. https://doi.org/10.1126/science.286.5437.77

Soderlund, K. M. (2019). Ocean dynamics of outer solar system satellites. Geophysical Research Letters, 46, 8700-8710. https://doi. org/10.1029/2018GL081880

Soderlund, K. M., Kalousová, K., Buffo, J. J., Glein, C. R., Goodman, J. C., Mitri, G., et al. (2020). Ice-ocean exchange processes in the jovian and Saturnian satellites. Space Science Reviews, 216(5), 1-57. https://doi.org/10.1007/s11214-020-00706-6

Soderlund, K. M., Schmidt, B. E., Wicht, J., \& Blankenship, D. D. (2014). Ocean-driven heating of Europa's icy shell at low latitudes. Nature Geoscience, 7, 16-19. https://doi.org/10.1038/ngeo2021

Srivastava, S. P. (1966). Theory of the magnetotelluric method for a spherical conductor. Geophysical Journal International, 11(4), $373-387$. https://doi.org/10.1111/j.1365-246x.1966.tb03090.x

Steinbrügge, G., Schroeder, D., Haynes, M., Hussmann, H., Grima, C., \& Blankenship, D. (2018). Assessing the potential for measuring Europa's tidal Love number h2 using radar sounder and topographic imager data. Earth and Planetary Science Letters, 482, 334-341. https://doi.org/10.1016/j.epsl.2017.11.028

Styczinski, M. J., \& Harnett, E. M. (2021). Induced magnetic moments from a nearly spherical ocean. Icarus, 354, 114020. https://doi. org/10.1016/j.icarus.2020.114020

Travis, B., Palguta, J., \& Schubert, G. (2012). A whole-moon thermal history model of Europa: Impact of hydrothermal circulation and salt transport. Icarus, 218, 1006-1019. https://doi.org/10.1016/j.icarus.2012.02.008

Tyler, R. H. (2011). Magnetic remote sensing of Europa's ocean tides. Icarus, 211, 906-908. https://doi.org/10.1016/j.icarus.2010.10.011

Tyler, R. H., Maus, S., \& Luhr, H. (2003). Satellite observations of magnetic fields due to ocean tidal flow. Science, 299, 239-241. https:// doi.org/10.1126/science.1078074

Vance, S., Bouffard, M., Choukroun, M., \& Sotin, C. (2014). Ganymede's internal structure including thermodynamics of magnesium sulfate oceans in contact with ice. Planetary and Space Science, 96, 62-70. https://doi.org/10.1016/j.pss.2014.03.011

Vance, S., \& Brown, J. (2005). Layering and double-diffusion style convection in Europa's ocean. Icarus, 177(2), 506-514. https://doi. org/10.1016/j.icarus.2005.06.005

Vance, S., \& Brown, J. (2013). Thermodynamic properties of aqueous $\mathrm{MgSO}_{4}$ to $800 \mathrm{MPa}$ at temperatures from -20 to $100^{\circ} \mathrm{C}$ and concentrations to $2.5 \mathrm{~mol} \mathrm{~kg}$ from sound speeds, with applications to icy world oceans. Geochimica et Cosmochimica Acta, 110, 176-189.

Vance, S., \& Goodman, J. (2009). Europa (p. 459-482). R. T. P. W. B. M. Khurana (Ed.). Arizona University Press.

Vance, S. D., Hand, K. P., \& Pappalardo, R. T. (2016). Geophysical controls of chemical disequilibria in Europa. Geophysical Research Letters, 43(10), 4871-4879. https://doi.org/10.1002/2016gl068547

Vance, S. D., Panning, M. P., Stähler, S., Cammarano, F., Bills, B. G., Tobie, G., et al. (2018). Geophysical investigations of habitability in ice-covered ocean worlds. Journal of Geophysical Research: Planets, 123, 180-205. https://doi.org/10.1002/2017je005341

Vance, S. D., Tobie, G., Daswani, M. M., \& Choblet, G. (2019). Tidal signatures of geochemically rigorous interior structure for Ganymede. EPSC Abstracts, 3(EPSC-DPS2019), 1149-1.

Zhu, P., Manucharyan, G. E., Thompson, A. F., Goodman, J. C., \& Vance, S. D. (2017). The influence of meridional ice transport on Europa's ocean stratification and heat content. Geophysical Research Letters, 44(12), 5969-5977. https://doi.org/10.1002/2017gl072996

Zimmer, C., Khurana, K. K., \& Kivelson, M. G. (2000). Subsurface oceans on Europa and Callisto: Constraints from Galileo magnetometer observations. Icarus, 147(2), 329-347. https://doi.org/10.1006/icar.2000.6456

Zolotov, M. (2008). Oceanic composition on europa: Constraints from mineral solubilities. In Lunar and Planetary Institute Science Conference Abstracts (Vol. 39, p. 2349).

Zolotov, M. Y., \& Kargel, J. (2009). On the chemical composition of Europa's icy shell, ocean, and underlying rocks. In R. T. Pappalardo, W. B. McKinnon, \& K. Khurana (Eds.), Europa (pp. 431-458). Tucson, AZ: University of Arizona Press.

Zolotov, M., \& Shock, E. (2001). Composition and stability of salts on the surface of Europa and their oceanic origin. Journal of Geophysical Research, 106(E12), 32815-32828. https://doi.org/10.1029/2000JE001413 